\title{
Comparative Study of Several Methods for the Calculation of Ultrasonic Guided Waves in Composites
}

\author{
Ayman $\mathrm{Kamal}^{1}$, Matthieu Gresil ${ }^{2}$ and Victor Giurgiutiu ${ }^{3}$ \\ LAMSS, University of South Carolina, Columbia, SC, 29208
}

\begin{abstract}
The objective of this study is studying the different predictive models for evaluating dispersion curves of ultrasonic guided waves in composites. The paper reviews different algorithms used for wave propagation analysis in layered composite plates: (a) Transfer Matrix (TM), (b) Global Matrix (GM), (c) Semi-Analytical Finite Element method (SAFE), (d) local interaction simulation approach (LISA), and (e) Equivalent Matrix (EM) method applied on cross-ply cases. Description of each technique is covered, then, advantages and distinct features of those techniques are presented. Case studies for unidirectional, cross ply, and general quasi isotropic laminates are presented. Finally, the paper ends by experimental and finite element simulation studies on glass fiber reinforced polymer composites (GFRP).
\end{abstract}

\section{Nomenclature}

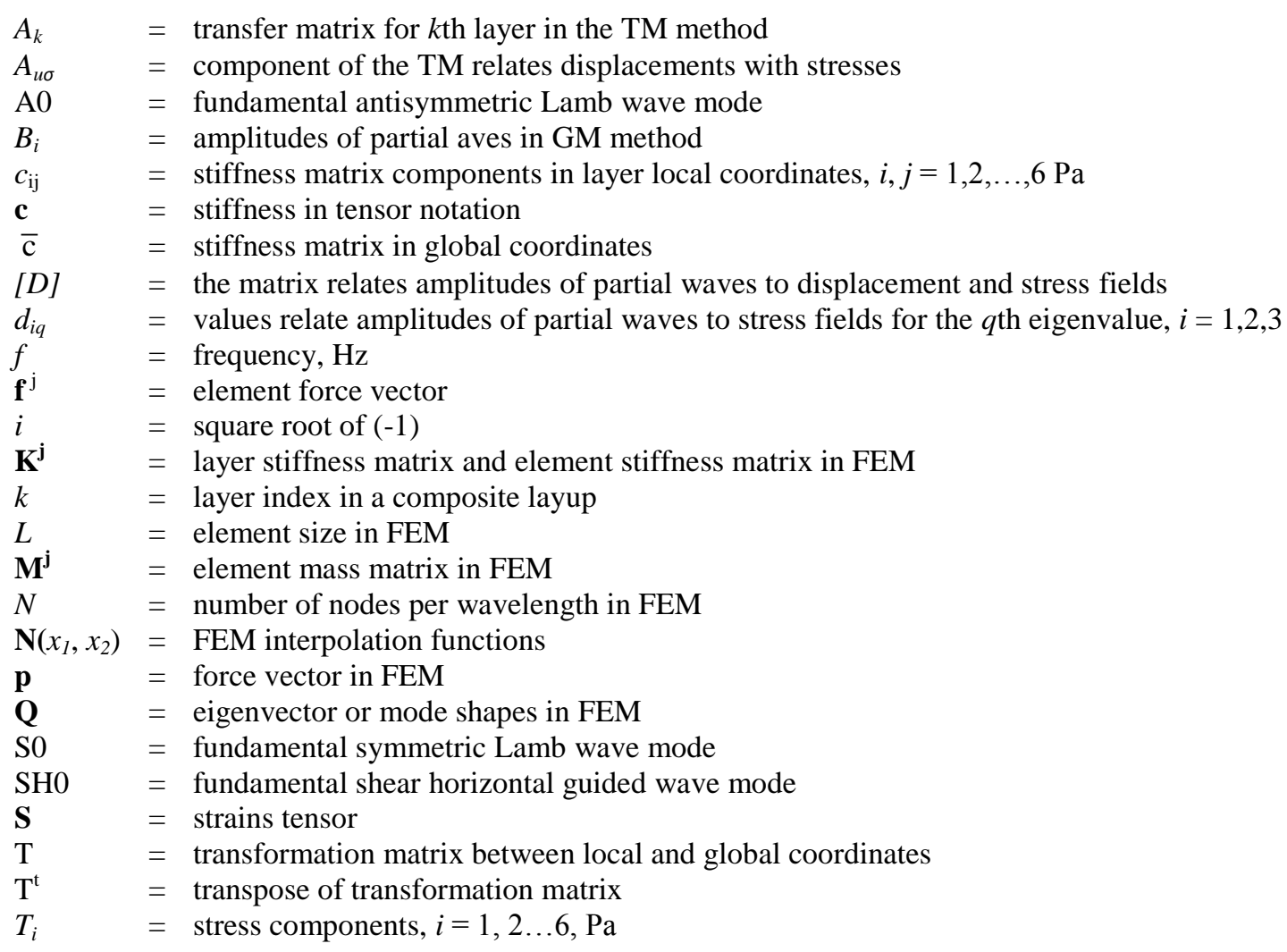

\footnotetext{
${ }^{1}$ Ph.D. student, Department of Mechanical Engineering University of South Carolina, 300 Main St. Columbia, SC 29208. Tel: 803-777-1535 Fax: 803-777-0106; kamal@email.sc.edu

${ }^{2}$ Research Associate, Department of Mechanical Engineering University of South Carolina, 300 Main St. Columbia, SC 29208. Tel: 803-777-0619 Fax: 803-777-0106; matthieu @cec.sc.edu

${ }^{3}$ Professor, Department of Mechanical Engineering University of South Carolina, 300 Main St. Columbia, SC

29208. AIAA Associate Fellow. Tel: 803-777-8018 Fax: 803-777-0106; victorg@sc.edu
} 


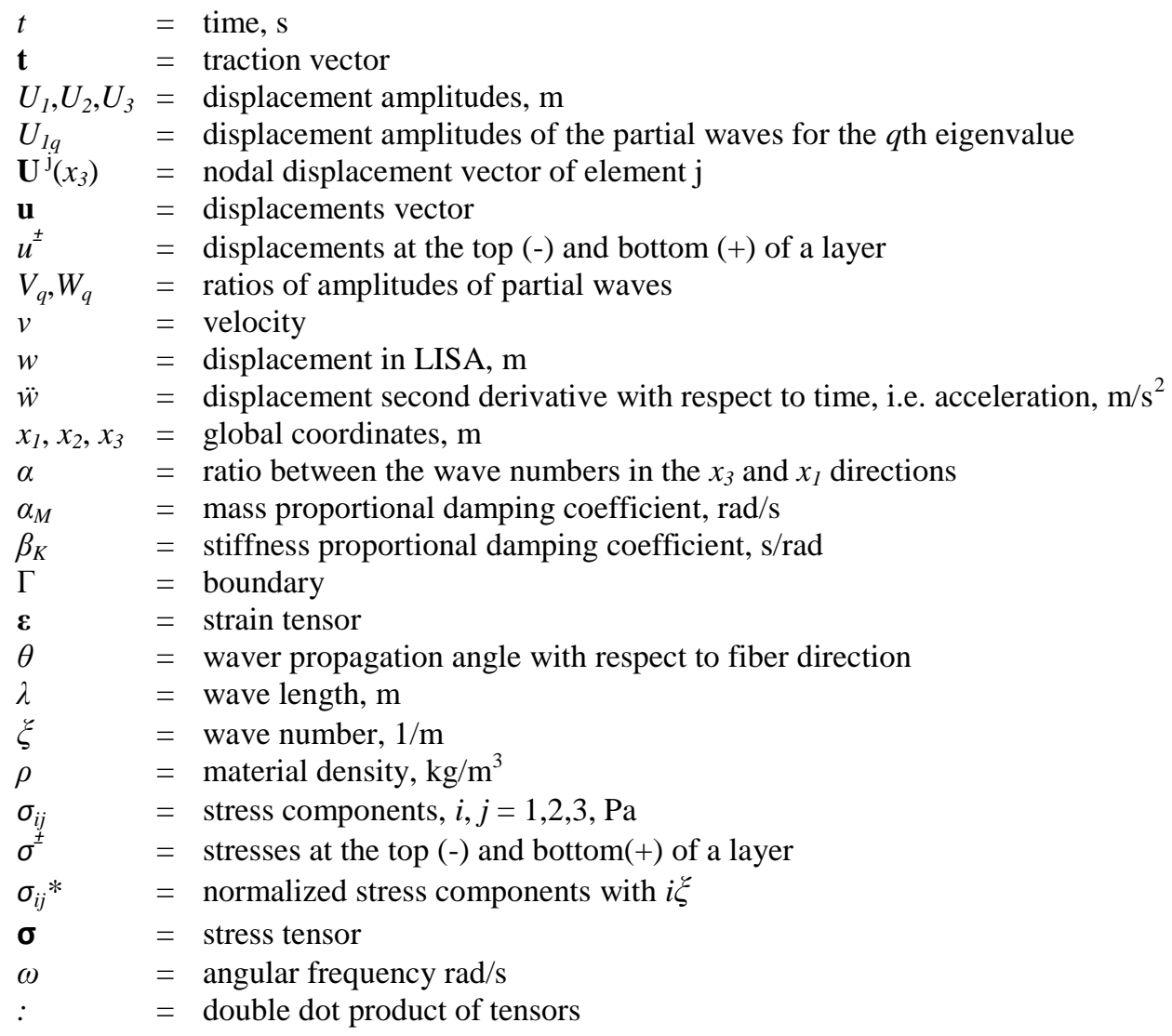

\section{Introduction}

$\mathrm{T}$

HE use of composite materials is currently implemented in many structural components, including automotive parts, civil infrastructures, compensatory devices and aerospace structures. Composite materials combine the properties of two or more constituent materials, for example, carbon-fiber reinforced polymer composites (CFRP) combine the specific stiffness and strength of carbon fibers with the properties of epoxy matrix. Composite materials can be generally manufactured from metallic, polymeric, or ceramic matrix; however, in this paper the focus is on polymer matrix composites for their wide application in the aerospace industry. Many parts of recent air and spacecraft are manufactured from CFRP and glass-fiber reinforced polymers (GFRP) as well. The need of lightweight, yet strong structural parts makes polymer composites more favorable than metallic alloys. Also, polymer composites can be manufactured into complex shaped components and their properties can be tailored by changing the stacking sequence of layup, i.e. layers or individual lamina.

Detection of damages and flaws as well as structural integrity of polymer composites is receiving as much attention as the advantages and applications of these materials. Lamb waves ultrasonics, or guided plate waves have long been acknowledged for damage detection in composites [1,2,3]. For any study of guided waves propagation in structures, wave's propagation speeds are essential for further analysis, e.g. impact source localization, reflection, transmission and mode conversion at damages. In many cases, robust predictive models of wave's speeds are needed before conducting experimental studies. Therefore; in this paper, our focus on comparing different methods of calculating ultrasonic guided wave's speeds in composite materials.

Lamb wave theory is well documented in many references $[1,4,5,6]$. For isotropic materials, the wave equation can be expressed by two potential functions and the pressure and shear wave velocities. The shear horizontal (SH) wave propagation in this case is decoupled from longitudinal (or pressure waves P) and shear vertical (SV) wave propagations. Lamb waves are symmetric and antisymmetric and they are dispersive by nature, i.e. (are having different speeds at different frequencies). The characteristic equation (Rayleigh-Lamb equation) is obtained by solving are equation and apply stress free boundary conditions at upper and lower surfaces of the plate.

In the case of fiber reinforced polymer (FRP) composites where the material is generally anisotropic, the three types of guided waves $(\mathrm{P}, \mathrm{SV}$, and $\mathrm{SH}$ ) are coupled and it is not possible to find close form solution of the dispersion curves. There are different methods to calculate dispersion curves in multilayered composite materials (a) Transfer 
Matrix method (TM); (b) Global Matrix method (GM); (c) Semi-Analytical Finite Element method (SAFE); (d) local interaction simulation approach (LISA); and (e) Equivalent Matrix method (EM) applied on cross-ply cases. Mathematical formulations of those techniques are presented. Case studies for unidirectional; cross ply; and general quasi isotropic laminates are compared using those methods. And the paper ends by experimental and finite element method simulation results.

\section{Algorithms for Calculating Dispersion Curves in Composites}

In this section, we show description and mathematical formulation of the methods used for calculating dispersion curves in FRP composites.

\section{A. Transfer Matrix method (TM)}

Transfer matrix method $[7,8]$ is a reliable technique for wave propagation analysis in layered media ; its advantage is that it condenses the multi-layered system into four equations (for the case of decoupled SH waves) or six equations relating the boundary conditions at the first and the last interfaces. It eliminates all other intermediate interfaces; which save a lot in terms of computational time and complexity. Hence, TM method is favorable. One drawback TM method suffers is its numerical instability of the solution at large frequency-thickness products [9].We follow the formulation in references $[10,11]$. Considering the composite plate layer is in $x_{1}, x_{2}$ plane with wave propagation along $x_{1}$ direction. And the angle of fibers with respect to direction of wave propagation is $\theta$. The layer stiffness matrix in global coordinates is

$$
[\bar{c}]=[T]^{-1}[c][T]^{-t}
$$

Where $T$ is the transformation matrix and can be found from many composites textbooks, e.g. [12]. And $c$ is the layer stiffness matrix in local coordinates. The maximum anisotropy we are considering is orthotropic layer; once the layer is rotated by angle $\theta$, it becomes monoclinic anisotropy. Equation of motion is

$$
\left\{\begin{array}{l}
\nabla \cdot\left(\mathbf{c}: \nabla_{s} \mathbf{u}\right)=\rho \frac{\partial^{2} \mathbf{u}}{\partial t^{2}} \\
\nabla_{s} \mathbf{u}=\mathbf{S}
\end{array}\right.
$$

Where $\rho$ is the densit, $\mathbf{S}$ is the strains tensor, $\mathbf{u}$ is the displacements vector and is decomposed into the three axes as

$$
\left(u_{1}, u_{2}, u_{3}\right)=\left(U_{1}, U_{2}, U_{3}\right) e^{i \xi\left(x_{1}+\alpha x_{3}-v t\right)}
$$

Where $\xi$ is the wave number in the $x_{1}$ direction, $v=\omega / \xi$ is the phase velocity, $\omega$ is the angular frequency, $\alpha$ is the ratio between wave number in the thickness direction $x_{3}$ and $x_{1}$ direction, and $U_{i}$ is the displacement amplitude. Substituting Eq. (3) into equation of motion, Eq. (2) and cancelling the exponential terms yields

$$
\left\{\begin{array}{l}
\left(c_{11}+c_{55} \alpha^{2}-\rho v^{2}\right) U_{1}+\left(c_{16}+c_{45} \alpha^{2}\right) U_{2}+\left(c_{13}+c_{55}\right) \alpha U_{3}=0 \\
\left(c_{16}+c_{45} \alpha^{2}\right) U_{1}+\left(c_{66}+c_{44} \alpha^{2}-\rho v^{2}\right) U_{2}+\left(c_{45}+c_{36}\right) \alpha U_{3}=0 \\
\left(c_{13}+c_{55}\right) \alpha U_{1}+\left(c_{36}+c_{45}\right) \alpha U_{2}+\left(c_{55}+c_{33} \alpha^{2}-\rho v^{2}\right) U_{3}=0
\end{array}\right.
$$

This is an eigenvalue problem, and the determinant of Eq. (4) can be expressed as

$$
\alpha^{6}+A_{1} \alpha^{4}+A_{2} \alpha^{2}+A_{3}=0
$$

Where $A_{i}$ values are found in Ref. [10] with slight corrections as

$$
\begin{aligned}
A_{1}= & {\left[c_{11} c_{33} c_{44}-c_{13}^{2} c_{44}+2 c_{13} c_{36} c_{45}-2 c_{13} c_{44} c_{55}+2 c_{13} c_{45}^{2}-2 c_{16} c_{33} c_{45}+c_{33} c_{55} c_{66}-c_{36}^{2} c_{55}\right.} \\
& \left.-\left(c_{33} c_{44}+c_{33} c_{55}+c_{44} c_{55}-c_{45}^{2}\right) \rho v^{2}\right] / \Delta \\
A_{2}= & {\left[c_{11} c_{33} c_{66}-c_{11} c_{36}^{2}-2 c_{11} c_{36} c_{45}+c_{11} c_{44} c_{55}-c_{11} c_{45}^{2}-c_{13}^{2} c_{66}+2 c_{13} c_{16} c_{36}+2 c_{13} c_{16} c_{45}-2 c_{13} c_{55} c_{66}\right.} \\
& -c_{16}^{2} c_{33}+2 c_{16} c_{36} c_{55} \\
& -\left(c_{11} c_{33}+c_{11} c_{44}-c_{13}^{2}-2 c_{13} c_{55}-2 c_{16} c_{45}+c_{33} c_{66}-c_{36}^{2}-2 c_{36} c_{45}+c_{44} c_{55}-c_{45}^{2}+c_{55} c_{66}\right) \rho v^{2} \\
& \left.+\left(c_{33}+c_{44}+c_{55}\right) \rho^{2} v^{4}\right] / \Delta \\
A_{3}= & {\left[c_{11} c_{55} c_{66}-c_{16}^{2} c_{55}-\left(c_{11} c_{55}+c_{11} c_{66}-c_{16}^{2}+c_{55} c_{66}\right) \rho v^{2}+\left(c_{11}+c_{55}+c_{66}\right) \rho^{2} v^{4}-\rho^{3} v^{6}\right] / \Delta } \\
\Delta= & c_{33} c_{44} c_{55}-c_{33} c_{45}^{2}
\end{aligned}
$$


By solving Eq. (5) symbolically; it can be shown that the eigenvalues $\alpha_{i}$ are in pairs, i.e.

$$
\alpha_{2}=-\alpha_{1}, \alpha_{4}=-\alpha_{3}, \alpha_{6}=-\alpha_{5}
$$

Using any two equations in Eq.(4), we find the displacements ratios (i.e. eigenvectors). However, careful selection of the two equations is important. If this algorithm is used for isotropic metallic layer or a composite layer that is almost isotropic, the displacements ratio $W_{q}=U_{3 q} / U_{1 q}$ suffers a singularity situation. Therefore, the ratios documented in references $[10,11]$ are exchanged by [13]

$$
\left\{\begin{array}{l}
V_{q}=\frac{U_{2 q}}{U_{1 q}}=\frac{\left(c_{11}+c_{55} \alpha_{q}^{2}-\rho v^{2}\right)\left(c_{45}+c_{36}\right) \alpha_{q}-\left(c_{16}+c_{45} \alpha_{q}^{2}\right)\left(c_{13}+c_{55}\right) \alpha_{q}}{\left(c_{13}+c_{55}\right) \alpha_{q}\left(c_{66}+c_{44} \alpha_{q}^{2}-\rho v^{2}\right)-\left(c_{16}+c_{45} \alpha_{q}^{2}\right)\left(c_{45}+c_{36}\right) \alpha_{q}} \\
W_{q}=\frac{U_{3 q}}{U_{1 q}}=\frac{\left(c_{11}+c_{55} \alpha_{q}^{2}-\rho v^{2}\right)\left(c_{66}+c_{44} \alpha_{q}^{2}-\rho v^{2}\right)-\left(c_{16}+c_{45} \alpha_{q}^{2}\right)^{2}}{\left(c_{16}+c_{45} \alpha_{q}^{2}\right)\left(c_{45}+c_{36}\right) \alpha_{q}-\left(c_{13}+c_{55}\right) \alpha_{q}\left(c_{66}+c_{44} \alpha_{q}^{2}-\rho v^{2}\right)}
\end{array}\right.
$$

Where $q$ represents the solution number from one to six. By doing this, a more general robust algorithm is attained. The displacements in Eq. (3) can be written as

$$
\left(u_{1}, u_{2}, u_{3}\right)=\sum_{q=1}^{6}\left(1, V_{q}, W_{q}\right) U_{1 q} e^{i \xi\left(x_{1}+\alpha_{q} x_{3}-v t\right)}
$$

And the stresses are

$$
\left\{\begin{array}{c}
T_{1} \\
T_{2} \\
T_{3} \\
T_{4} \\
T_{5} \\
T_{6}
\end{array}\right\}=i \xi \sum_{q}\left\{\begin{array}{c}
c_{11}+\alpha_{q} c_{13} W_{q}+c_{16} V_{q} \\
c_{12}+\alpha_{q} c_{23} W_{q}+c_{26} V_{q} \\
c_{13}+\alpha_{q} c_{33} W_{q}+c_{36} V_{q} \\
\alpha_{q} c_{44} V_{q}+c_{45}\left(\alpha_{q}+W_{q}\right) \\
\alpha_{q} c_{45} V_{q}+c_{55}\left(\alpha_{q}+W_{q}\right) \\
c_{16}+\alpha_{q} c_{36} W_{q}+c_{66} V_{q}
\end{array}\right\} U_{1 q} e^{i \xi\left(x_{1}+\alpha_{q} x_{3}-v t\right)}
$$

The stresses that are of interest are $\sigma_{33}, \sigma_{13}, \sigma_{23}$. Stress free boundary condition is applied on them, define

$$
\left(\sigma_{33}^{*}, \sigma_{13}^{*}, \sigma_{23}^{*}\right)=\left(T_{3}^{*}, T_{5}^{*}, T_{4}^{*}\right)=\sum_{q=1}^{6}\left(d_{1 q}, d_{2 q}, d_{3 q}\right) U_{1 q} e^{i \xi\left(x_{1}+\alpha_{q} x_{3}-v t\right)}
$$

Where $\sigma^{*}=\sigma / i \xi$, and $d_{1 q}, d_{2 q}, d_{3 q}$ are contracted terms from Eq. (10).

The relation for wave propagation in a layer with combining displacement and stress relations is

$$
\left[\begin{array}{l}
u_{1} \\
u_{2} \\
u_{3} \\
\sigma_{33}^{*} \\
\sigma_{13}^{*} \\
\sigma_{23}^{*}
\end{array}\right]=\left[\begin{array}{cccccc}
1 & 1 & 1 & 1 & 1 & 1 \\
V_{1} & V_{1} & V_{3} & V_{3} & V_{5} & V_{5} \\
W_{1} & -W_{1} & W_{3} & -W_{3} & W_{5} & -W_{5} \\
d_{11} & d_{11} & d_{13} & d_{13} & d_{15} & d_{15} \\
d_{21} & -d_{21} & d_{23} & -d_{23} & d_{25} & -d_{25} \\
d_{31} & -d_{31} & d_{31} & -d_{31} & d_{35} & -d_{35}
\end{array}\right]\left[\begin{array}{c}
U_{11} e^{i \xi \alpha_{1} x_{3}} \\
U_{11} e^{-i \xi \alpha_{1} x_{3}} \\
U_{13} e^{i \xi \alpha_{3} x_{3}} \\
U_{13} e^{-i \xi \alpha_{3} x_{3}} \\
U_{15} e^{i \xi \alpha_{5} x_{3}} \\
U_{15} e^{-i \xi \alpha_{5} x_{3}}
\end{array}\right] e^{i \xi\left(x_{1}-v t\right)}
$$

The idea of TM method is relating the layer properties and boundary conditions at the top and bottom surfaces with other layers. This is done by applying continuity of displacements and equilibrium of stresses. The layer transfer matrix $A_{k}$ relates the displacements and stresses of the top of the layer to those of the bottom of the layer

$$
\left\{\begin{array}{l}
\left\{u^{+}\right\} \\
\left\{\sigma^{+}\right\}
\end{array}\right\}=\left[\begin{array}{ll}
{\left[A_{u u}\right]} & {\left[A_{u \sigma}\right]} \\
{\left[A_{u \sigma}\right]} & {\left[A_{\sigma \sigma}\right]}
\end{array}\right]\left\{\begin{array}{l}
\left\{u^{-}\right\} \\
\left\{\sigma^{-}\right\}
\end{array}\right\}
$$

Where $A_{k}$ is the $4 \times 4$ matrix of Eq. (13). Call the $6 \times 6$ matrix of Eq. (12) $X$, the vector of $U_{1 i}$ elements $U$, and the diagonal matrix of elements $e^{i \xi_{i} \alpha_{3}} D$. Hence,

$$
A_{k}=X_{k} D_{k} X_{k}^{-1}
$$


The total TM is calculated by multiplying the transfer matrix of individual layers consecutively. And to satisfy stress free boundary condition for the whole laminate; $\sigma^{+}$and $\sigma^{-}$in Eq. (13) are set to zero, hence the characteristic equation to find dispersion phase velocities versus wavenumbers is

$$
\left|A_{u \sigma}\right|=0
$$

Usually Eq. (15) is solved numerically to find dispersion phase velocities versus wavenumbers or frequencies. This will be discussed in the GM section

\section{B. Stable Stiffness Transfer Matrix method (SSTM)}

Rokhlin [13] showed that due to refraction within one or more layers of the laminate; some of the plane waves can be internally reflected, meaning that their partial waves will be evanescent within the layer, i.e. propagating constant will be imaginary in the exponential $e^{ \pm i \xi x_{3}}$. Depending on the thickness and the frequency the real term $e^{\xi x_{3}}$ can be very large or very small. The TM formulation in itself has no deficiency. But numerical computation of this real exponential that rises and falls quickly suffers instabilities frequently (Figure 1).

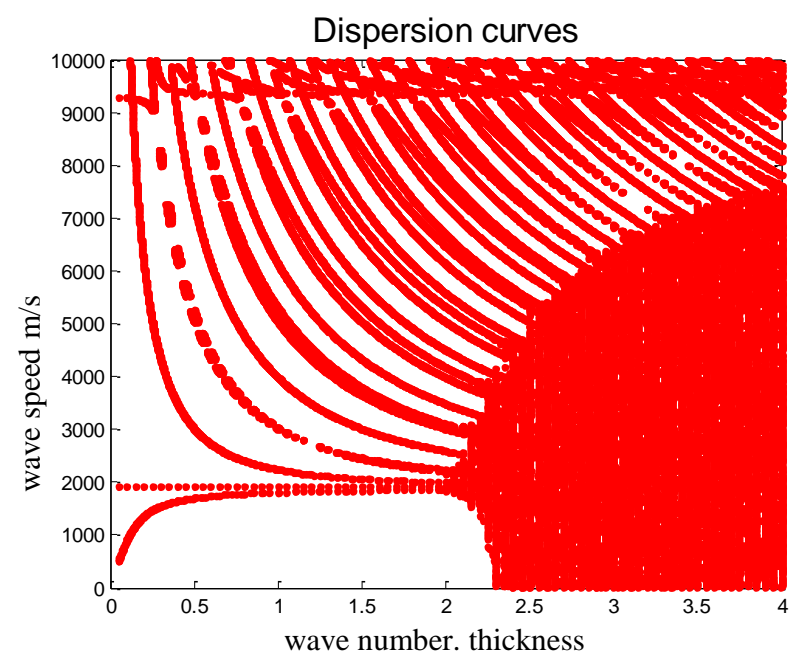

Figure 1. Instability of TM method.

There have been many publications proposing reformulation of equations to avoid this problem e.g. [14]. The method is based on using stiffness matrix (SM) instead of (TM); this is done by re-arranging terms of the TM such that displacements at both the top and the bottom of the $\mathrm{j}$ layer in a single column matrix. Similarly, tractions at both the top and the bottom of the $\mathrm{j}$ layer are combined in one single column matrix. The transfer function between them will be the stiffness matrix (SM) or $\mathbf{K}^{\mathbf{j}}$,

$$
\left[\begin{array}{c}
\sigma_{j-1} \\
\sigma_{j}
\end{array}\right]=\mathbf{K}^{\mathbf{j}}\left[\begin{array}{c}
u_{j-1} \\
u_{j}
\end{array}\right]
$$

This is on contrast to the transfer matrix in which displacement and traction for each boundary are combined in the one single column vector, Eq. (13). The recursive approach to find the "total" stiffness matrix of all layers is well documented in the Ref. [13]. That approach is not straight forward as the one applied for finding the total TM. For total TM, we simply multiply transfer matrix of all individual layers. The total stiffness matrix for two layers is [13]

$$
\left[\begin{array}{c}
\sigma_{0} \\
\sigma_{2}
\end{array}\right]=\left[\begin{array}{cc}
K_{11}^{A}+K_{12}^{A}\left(K_{11}^{B}-K_{22}^{A}\right)^{-1} K_{21}^{A} & -K_{12}^{A}\left(K_{11}^{B}-K_{22}^{A}\right)^{-1} K_{12}^{B} \\
K_{21}^{B}\left(K_{11}^{B}-K_{22}^{A}\right)^{-1} K_{21}^{A} & K_{22}^{B}-K_{21}^{B}\left(K_{11}^{B}-K_{22}^{A}\right)^{-1} K_{12}^{B}
\end{array}\right]\left[\begin{array}{l}
u_{0} \\
u_{2}
\end{array}\right]
$$

For complete understanding of that approach and implementation in Nayfeh formulation [10], recall the $X$ matrix in Eq.(12), and for simplicity, we consider the case of decoupled SH waves, where the $X$ matrix is reduced to $4 \times 4$ 


$$
\left[\begin{array}{c}
u_{1} \\
u_{3} \\
\sigma_{33}^{*} \\
\sigma_{13}^{*}
\end{array}\right]=\left[\begin{array}{cccc}
1 & 1 & 1 & 1 \\
W_{3} & -W_{3} & W_{5} & -W_{5} \\
d_{13} & d_{13} & d_{15} & d_{15} \\
d_{23} & -d_{23} & d_{25} & -d_{25}
\end{array}\right]\left[\begin{array}{cccc}
e^{i \xi \alpha_{3} x_{3}} & 0 & 0 & 0 \\
0 & e^{-i \xi \alpha_{3} x_{3}} & 0 & 0 \\
0 & 0 & e^{i \xi \alpha_{5} x_{3}} & 0 \\
0 & 0 & 0 & e^{-i \xi \alpha_{5} x_{3}}
\end{array}\right]\left[\begin{array}{c}
U_{13} \\
U_{13} \\
U_{15} \\
U_{15}
\end{array}\right] e^{i \xi\left(x_{1}-v t\right)}
$$

For the case of decoupled SH waves; $W_{3}, W_{5}$ can be explicitly expressed in terms of $\alpha_{3}, \alpha_{5}$ as [10]

$$
W_{3}=\alpha_{3}, W_{5}=-1 / \alpha_{5}
$$

Equation (18) is written in terms of Eq. (19) and $D_{k}$ values of Eq. (14)

$$
\left[\begin{array}{c}
u_{1} \\
u_{3} \\
\sigma_{33}^{*} \\
\sigma_{13}^{*}
\end{array}\right]=\left[\begin{array}{cccc}
1 & 1 & 1 & 1 \\
\alpha_{3} & -\alpha_{3} & -1 / \alpha_{5} & 1 / \alpha_{5} \\
d_{13} & d_{13} & d_{15} & d_{15} \\
d_{23} & -d_{23} & d_{25} & -d_{25}
\end{array}\right]\left[\begin{array}{cccc}
D_{1} & 0 & 0 & 0 \\
0 & D_{2} & 0 & 0 \\
0 & 0 & D_{3} & 0 \\
0 & 0 & 0 & D_{4}
\end{array}\right]\left[\begin{array}{l}
U_{13} \\
U_{13} \\
U_{15} \\
U_{15}
\end{array}\right]
$$

For the top surface (j-1) as shown in Figure 2; $x_{3}=0$ in the diagonal matrix $D_{k}$ of Eq. (20). This results to a unit matrix. For the bottom surface $(j)$, we use $D_{k}=e^{i \xi \alpha_{i} d}$, where $d$ is the layer thickness.

Equation (20) is redefined for both $\left[\begin{array}{l}u_{1}^{j-1} \\ u_{3}^{j-1} \\ \sigma_{33}^{j-1} \\ \sigma_{13}^{j-1}\end{array}\right]$ and $\left[\begin{array}{l}u_{1}^{j} \\ u_{3}^{j} \\ \sigma_{33}^{j} \\ \sigma_{13}^{j}\end{array}\right]$. The stiffness matrix is
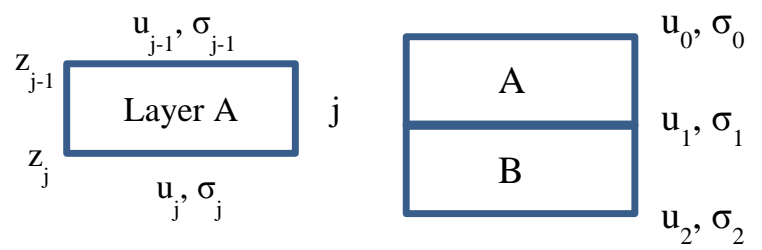

Figure 2. Notation of subscripts for displacements and stresses then constructed by combining all stresses at top and bottom of each layer.

terms in one vector and displacement terms in another,

$$
\begin{aligned}
& {\left[\begin{array}{c}
\sigma_{33}^{j-1} \\
\sigma_{13}^{j-1} \\
\sigma_{33}^{j} \\
\sigma_{13}^{j}
\end{array}\right]=\left[\begin{array}{cccc}
d_{13} & d_{13} & d_{15} & d_{15} \\
d_{23} & -d_{23} & d_{25} & -d_{25} \\
d_{13} D_{1} & d_{13} D_{2} & d_{15} D_{3} & d_{15} D_{4} \\
d_{23} D_{1} & -d_{23} D_{2} & d_{25} D_{3} & -d_{25} D_{4}
\end{array}\right]\left[\begin{array}{l}
U_{13} \\
U_{13} \\
U_{15} \\
U_{15}
\end{array}\right]} \\
& {\left[\begin{array}{c}
u_{1}^{j-1} \\
u_{3}^{j-1} \\
u_{1}^{j} \\
u_{3}^{j}
\end{array}\right]=\left[\begin{array}{cccc}
1 & 1 & 1 & 1 \\
\alpha_{3} & -\alpha_{3} & -1 / \alpha_{5} & 1 / \alpha_{5} \\
D_{1} & D_{2} & D_{3} & D_{4} \\
\alpha_{3} D_{1} & -\alpha_{3} D_{2} & -D_{3} / \alpha_{5} & D_{4} / \alpha_{5}
\end{array}\right]\left[\begin{array}{l}
U_{13} \\
U_{13} \\
U_{15} \\
U_{15}
\end{array}\right]}
\end{aligned}
$$

Define the $4 \times 4$ matrix in Eq. (21) as $Y$, and the $4 \times 4$ matrix of Eq. (22) as $Y_{2}$; The layer SM is determined from

$$
\left(\begin{array}{c}
\boldsymbol{\sigma}_{x_{3}=0} \\
\boldsymbol{\sigma}_{x_{3}=-d}
\end{array}\right)=Y . Y_{2}^{-1} \cdot\left(\begin{array}{c}
\mathbf{u}_{x_{3}=0} \\
\mathbf{u}_{x_{3}=-d}
\end{array}\right)
$$

Where $\mathbf{S M}=\mathbf{Y} . \mathbf{Y}^{-1}$. Then, the total SM as reported in Ref. [13] and Eq. (17) is achieved by recursive algorithm. For the sake of implementation into Nayfeh [10] formulation; we constructed a flow chart of Rokhlin algorithm (Figure $3)$. The $\mathrm{SM}$ for individual layers $\mathrm{A}$ and $\mathrm{B}$ are defined as

$$
\left[\begin{array}{l}
\sigma_{0} \\
\sigma_{1}
\end{array}\right]=\left[\begin{array}{ll}
K_{11}^{A} & K_{12}^{A} \\
K_{21}^{A} & K_{22}^{A}
\end{array}\right]\left[\begin{array}{l}
u_{0} \\
u_{1}
\end{array}\right] \quad, \quad\left[\begin{array}{l}
\sigma_{1} \\
\sigma_{2}
\end{array}\right]=\left[\begin{array}{ll}
K_{11}^{B} & K_{12}^{B} \\
K_{21}^{B} & K_{22}^{B}
\end{array}\right]\left[\begin{array}{l}
u_{1} \\
u_{2}
\end{array}\right]
$$




\section{Global Matrix method (GM)}

Global matrix is first developed by [15]. It is combining stresses and displacements at the boundaries of each layer with boundary conditions and assembles them in one single matrix. Compared to Thomson-Haskell $[8,7]$ transfer matrix technique; global matrix has the advantage that it remains stable at high frequency-thickness products. GM shows the same base matrix whatever for real or imaginary wave numbers, vacuum, liquid, or solid halfspace [9]. The disadvantage is that the global matrix end to be a large matrix for laminates with large numbers of layers. Following is a brief description of the method [16].

In the GM method, one matrix represents the whole system, and if $\mathrm{SH}$ waves are not decoupled; then the general size of the matrix is $6(n-1)$ equations, where $n$ is the number of layers including the two semi-infinite media. Figure 4 shows the example of three layered material and the corresponding GM. For simplicity of showing the algorithm, SH waves are considered decoupled. The corresponding number of equations reduces to $4(n-1)$. The assembled equations

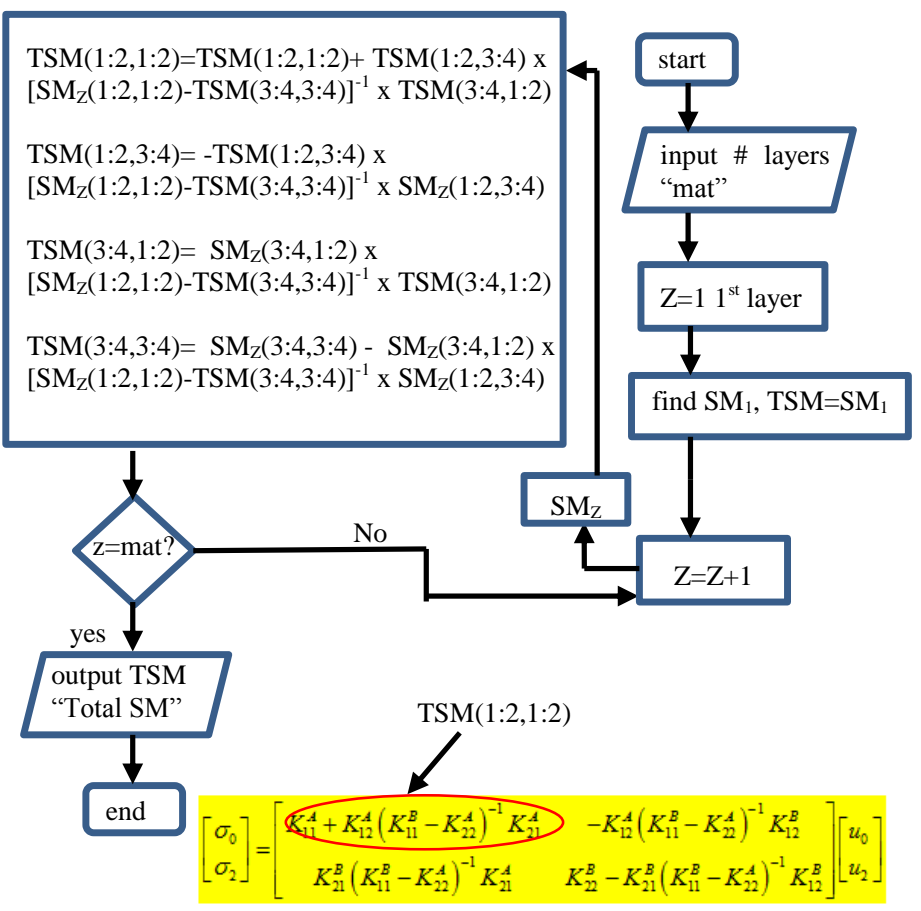

Figure 3. Flow chart of total SM recursive algorithm.

$$
\left[\begin{array}{cccc}
{\left[D_{1 b}^{-}\right]} & {\left[-D_{2 t}\right]} & & \\
& {\left[D_{2 b}\right]} & {\left[-D_{3 t}\right]} & \\
& {\left[D_{3 b}\right]} & {\left[-D_{4 t}\right]} \\
& & {\left[D_{4 b}\right]} & {\left[D_{5 b}^{+}\right]}
\end{array}\right]\left[\begin{array}{l}
B_{1} \\
B_{2} \\
B_{3} \\
B_{4} \\
B_{5}
\end{array}\right]=0
$$

Where $[D]$ is a $4 \times 4$ matrix that relates amplitude of the partial waves to the displacement and stress fields in a layer; $B_{i}$ is the amplitude of partial waves in layer $i ; t, b$ refer to top and bottom; +, - refer to waves going downwards or upwards (Figure 4).

Numerical solution for matrix methods

Root solving methods are mostly numerical and iterative; it was reported in $[9,16]$ that roots can be determined in a robust way by varying the phase velocity at fixed frequency or vice versa. Initial guess is needed and tracing is determined by linear extrapolation of two roots. Afterwards, when sufficient number of roots is determined; a quadratic extrapolation is used.
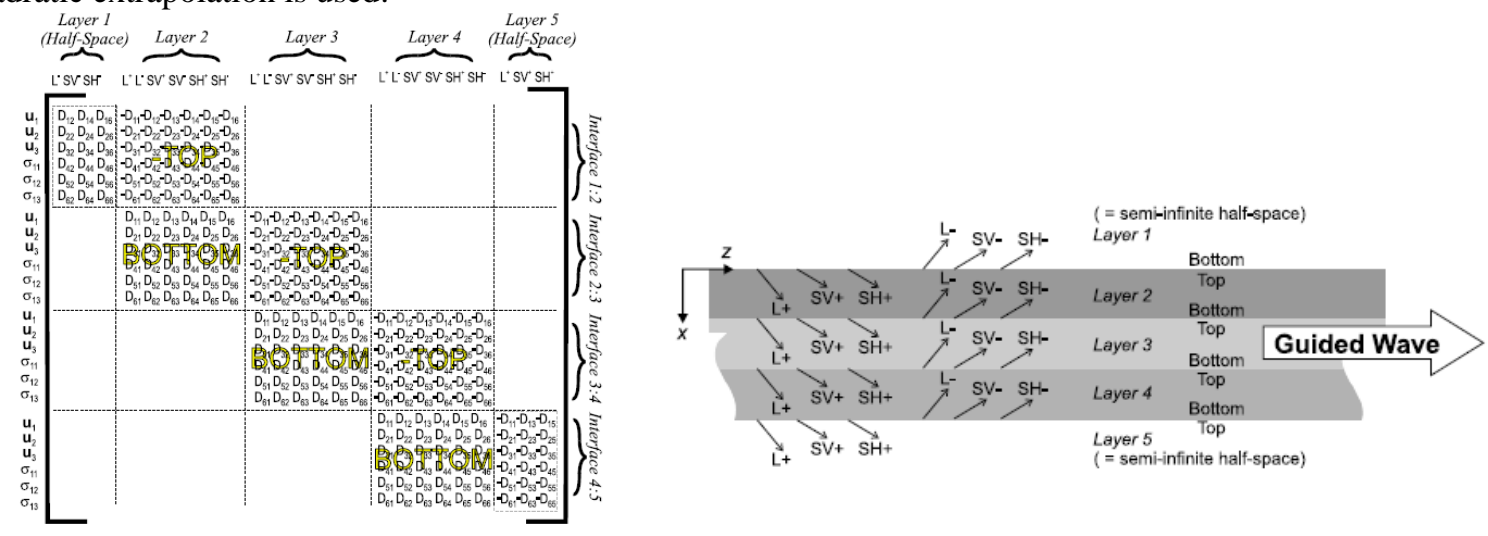

Figure 4. Global Matrix formulation [16].

American Institute of Aeronautics and Astronautics 


\section{Semi Analytical Finite Element method (SAFE)}

SAFE discretizes the structure cross section allowing different cross sections to be analyzed, because of the finite element discretizing in cross section. In the same time it solves analytically for wave propagation direction; which makes it more efficient in terms of computational time and memory than a complete FEM [17, 18]. The advantage of discretizing the cross section is that it allows the modeling of any arbitrary cross sections (e.g. rail [19, 20]). Also, in general the material is defined in FEM by stiffness matrix; that makes SAFE method to be very straight forward for application of anisotropic materials. SAFE is becoming popular for analyzing guided wave propagation in composites [20] SAFE solutions are obtained in a stable manner from an eigenvalue problem, and thus do not require the root-searching algorithms used in transfer matrix algorithms. A brief description following [19], the virtual work principle states

$$
\int_{\Gamma} \delta \mathbf{u}^{T} \mathbf{t} d \Gamma=\int_{V} \delta \mathbf{u}^{T}(\rho \ddot{\mathbf{u}}) d V+\int_{V} \delta \boldsymbol{\varepsilon}^{T} \boldsymbol{\sigma} d V
$$

Where ${ }^{T}$ is the transpose; $\rho$ is the density; $\int_{\Gamma}(). d \Gamma$ and $\int_{V}() d$.$V are the surface integration of the element and the$ volume integration of the element, respectively. $\int_{\Gamma} \delta \mathbf{u}^{T} \mathbf{t} d \Gamma$ denotes the work done by external traction $\mathbf{t}$. The two terms on right hand side of Eq. (26) are kinetic energy and potential energy. The wave solution along the wave propagation direction is represented by exponential orthogonal functions $\exp \left(i \xi_{i} x_{3}\right)$. The following step is similar to FEM discretization, where we define the displacement vector at arbitrary point

$$
\mathbf{u}=\mathbf{N}\left(x_{1}, x_{2}\right) \mathbf{U}^{j}\left(x_{3}\right) \exp (-i \omega t)
$$

Where $\mathbf{N}(x, y)$ is the interpolation is function, and $\mathbf{U}^{j}(z)$ is the nodal displacement vector of element $\mathrm{j}$. Strains are determined from the displacements and the derivatives of the interpolation functions with respect to $x_{1}, x_{2}$. Similar formulation is used for traction vector $\mathbf{t}$ in terms of nodal external traction vector. The stress vector is $\boldsymbol{\sigma}=c \boldsymbol{\varepsilon}$ in which material stiffness matrix is incorporated.

Substituting displacements, tractions, strains, and stresses in Eq. (26) yields

$$
\mathbf{f}^{j}=\left(\mathbf{K}_{1}^{j}+i \xi \mathbf{K}_{2}^{j}+\xi^{2} \mathbf{K}_{3}^{j}\right) \bar{U}^{j}-\omega^{2} \mathbf{M}^{j} \bar{U}^{j}
$$

Where $\mathbf{f}^{j}$ is the element force vector; $\mathbf{K}_{1-3}^{j}$ and $\mathbf{M}^{j}$ are the integrals determined by numerical integration techniques and are function of geometry; $\xi$ is the wave number; $\omega$ is the angular frequency. $\bar{U}^{j}$ is element nodal displacement vector. Common nodes results are overlapped and the total system governing equations are determined (without $j$ ). It is easy to introduce damping by considering complex form of the $\mathbf{K}$ matrices. The final governing equation [19, 20],

$$
\mathbf{A}=\left[\begin{array}{cc}
0 & \mathbf{A}-\xi \mathbf{B}) \mathbf{Q}=\mathbf{p} \\
\mathbf{K}_{1}-\omega^{2} \mathbf{M} & i \boldsymbol{K}_{2}^{2} \mathbf{M}
\end{array}\right], \quad \mathbf{B}=\left[\begin{array}{cc}
\mathbf{K}_{1}-\omega^{2} \mathbf{M} & 0 \\
0 & -\mathbf{K}_{3}
\end{array}\right], \quad \mathbf{Q}=\left[\begin{array}{c}
\overline{\mathbf{U}} \\
\xi \overline{\mathbf{U}}
\end{array}\right], \quad \mathbf{p}=\left[\begin{array}{l}
0 \\
\mathbf{f}
\end{array}\right]
$$

When the force vector $\mathbf{p}=0$; the eigenvalues $\xi_{m}$ of the system can be determined and the phase velocity are given by $c_{m}=\omega / \xi_{m}$. Consequently; nodal solutions can be determined (and the mode shapes).

\section{E. Local Interaction Simulation Approach (LISA)}

LISA discretizes the system into a lattice like finite difference method and its formulation is based on elastodynamic equations. The advantage of LISA appears when discontinuities or changes needed to be applied to the material properties; those changes are treated by simply modifying the properties of the lattice at the corresponding locations. LISA is well studied by Delsanto [21] for 3-D case, starting from the elastodynamic wave equation

$$
\partial_{l}\left(S_{k l m n} w_{m, n}\right)=\rho \ddot{w}_{k} \quad(k, l, m, n=1,3)
$$

Where $S$ is the stiffness tensor, $\rho$ is the material density, $w$ is displacement. Time is discretized and the propagation medium is discretized into a lattice with special steps [22]. Finite difference (FD) formulation is used in recursive equations to represent the second order space derivatives of the neighboring points of a generic point $\mathrm{O}$ in the lattice. 
The finite difference formalism is then used with the elastodynamic equation of motion to generate three iterative equations which allow computation of displacements $u_{p}$ at time $t+1$ as a linear combination of displacement components at time $t$ and $t-1$. A well-organized formulation was reported in Ref. [23] with experimental validations of LISA for both isotropic and anisotropic media. Another study [22] compares LISA approach with experimental results using laser vibrometer for elastic plates.

\section{F. Equivalent Matrix Method (EM)}

At the end more insight was put onto EM method; it is a very quick and reliable approach for analyzing cross ply laminates due to the fact that transformation matrix between 0 and 90 degrees is straight forward and can be done manually It can be applied for generally orientated layers as well; though the developed code in this study is only for cross ply laminates. The procedure is as follows

$$
\begin{aligned}
& {\left[S_{\text {uni }}\right]=\left[C_{\text {uni }}\right]^{-1}} \\
& {\left[S_{w}\right]=[T]^{t}\left[S_{\text {fill }}\right][T]}
\end{aligned}
$$

Where $S$ is the compliance matrix, and $C$ is the stiffness matrix, $T$ is the transformation matrix. Fill direction is the unidirectional one, and warp is the perpendicular one. Then the corresponding warp stiffness is calculated and the average is determined

$$
\begin{aligned}
& {\left[C_{w}\right]=\left[S_{w}\right]^{-1}} \\
& {\left[C_{e q}\right]=\left(C_{u n i}+C_{w}\right) / 2}
\end{aligned}
$$

This method is used in Ref. [24]. The example used in this study is T300/914 CFRP and the corresponding unidirectional lamina stiffness coefficients and the EM are

$$
C_{u n i}=\left(\begin{array}{cccccc}
143.8 & 6.2 & 6.2 & 0 & 0 & 0 \\
6.2 & 13.3 & 6.5 & 0 & 0 & 0 \\
6.2 & 6.5 & 13.3 & 0 & 0 & 0 \\
0 & 0 & 0 & 3.6 & 0 & 0 \\
0 & 0 & 0 & 0 & 5.7 & 0 \\
0 & 0 & 0 & 0 & 0 & 5.7
\end{array}\right) G P a, \quad C_{e q}=\left(\begin{array}{cccccc}
78.5 & 6.2 & 6.35 & 0 & 0 & 0 \\
6.2 & 78.5 & 6.5 & 0 & 0 & 0 \\
6.35 & 6.5 & 13.3 & 0 & 0 & 0 \\
0 & 0 & 0 & 4.65 & 0 & 0 \\
0 & 0 & 0 & 0 & 4.65 & 0 \\
0 & 0 & 0 & 0 & 0 & 5.7
\end{array}\right)
$$

Transformation matrix is available in many mechanics of composites textbooks, e.g. [12].

\section{Results and Comparisons}

In this section, results are shown for dispersion curves (phase velocities, and some group velocity curves), the material used as case study is T300/914 CFRP used in DISPERSE software manual [16] and other studies [20, 11]. The unidirectional layer stiffness matrix values are the values of $C_{u n i}$ in Eq. (33), the density is $\rho=1560 \mathrm{~kg} / \mathrm{m}^{3}$. These material properties are used for unidirectional case studies as well as cross ply cases, i.e. subsections A, B. DISPERSE software is used for GM method (with both phase and group velocity curves). GUIGUW computer package which is used in [20] and is available online is used for SAFE method; the core code is used with help from the developers instead of the online interface. Both TM method and EM results are developed by our group. SSTM method is still under development. And finally results reported for LISA are reported from literature [25] as we did not find yet an available tool based on LISA. 


\section{A. Unidirectional fiber laminated composites along different angles}

Table 1. T300/914 unidirectional fiber laminate with fiber along wave propagation direction $(\theta=0)$

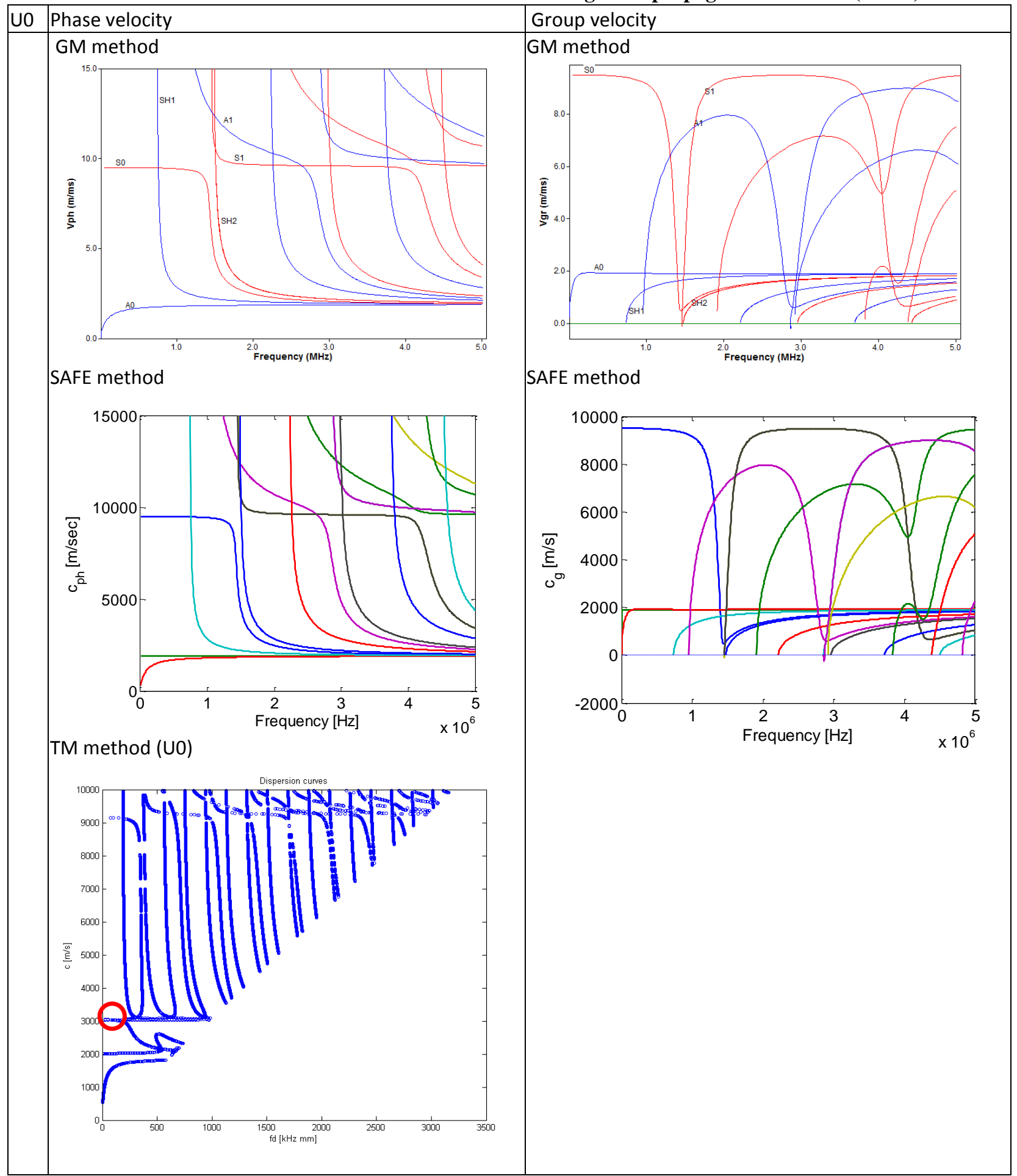

SAFE and GM dispersion curves of Table 1 are exactly reported in [16] pp.155 and [20] pp.698. Both methods GM, and SAFE are matching pretty well. From TM method in Table 1, more modes are compressed within the frequency axis. $\mathrm{S}_{0}$ mode has the initial speed around $9000 \mathrm{~m} / \mathrm{s}$. $\mathrm{SH}_{0}$ mode has the speed of $2000 \mathrm{~m} / \mathrm{s}$. The fact half of 
TM method dispersion curves is a half figure, because we did not run sufficient wave number- speed sweep to cover the frequency domain when we convert $\xi d$ into $f d$ domain.

Table 2. T300/914 unidirectional fiber laminate $(\theta=30)$

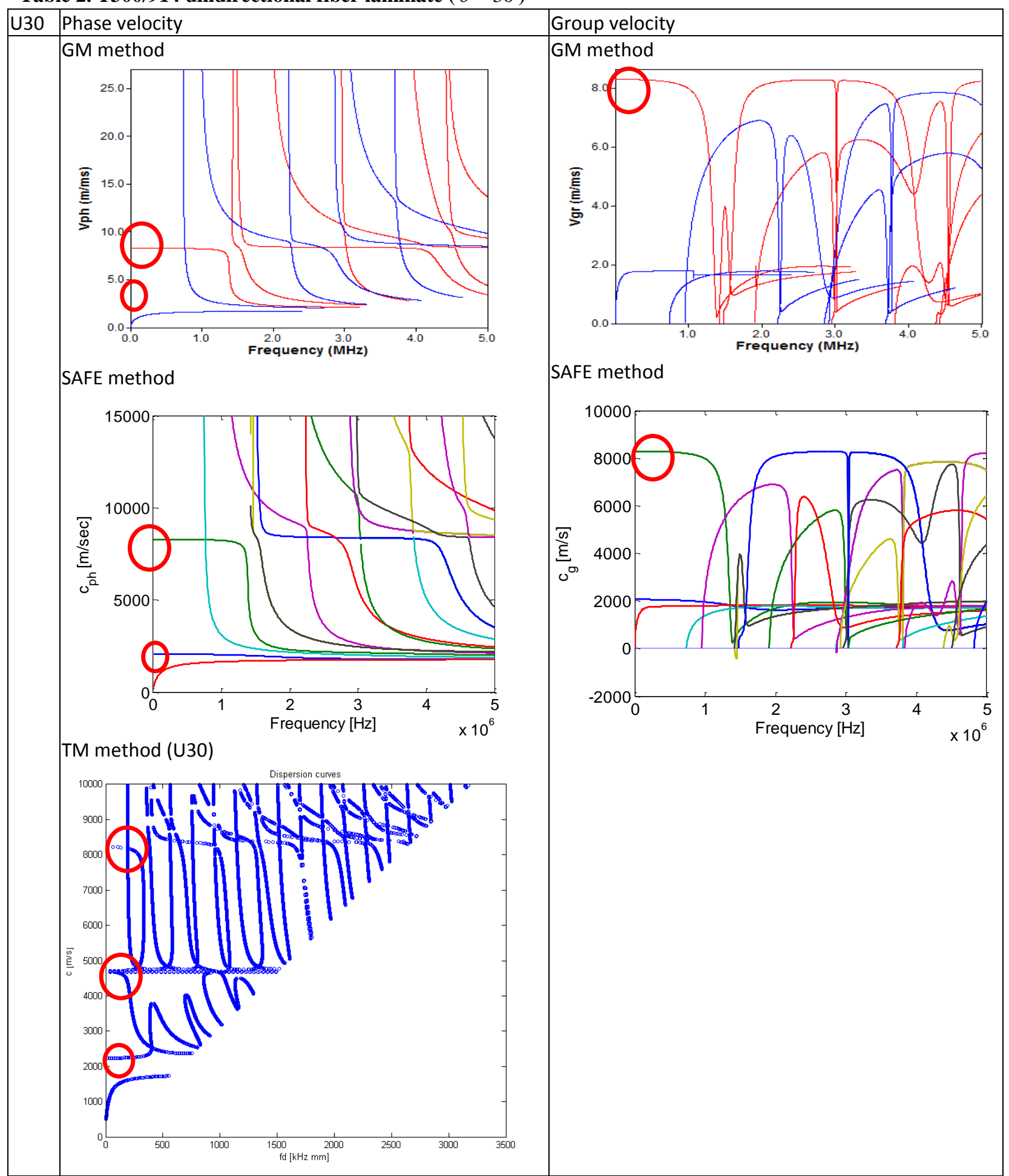

One observation (Table 2) is that SH0 sometimes are missed during DISPERSE root finding, but can be carefully found by manual sweep. As expected, as the angle increases between wave propagation direction and the fiber; the

11

American Institute of Aeronautics and Astronautics 
material is less stiff along U0 direction; and the wave speed is decreased. This behavior is reported by three methods, GM, SAFE and TM; as the dispersion phase velocity of $S_{0}$ becomes around $8000 \mathrm{~m} / \mathrm{s}$. However, for TM method, we always see the "mistake" root around 4000 to $5000 \mathrm{~m} / \mathrm{s}$. Next is the interesting 45 degrees unidirectional fiber case.

Table 3. T300/914 unidirectional fiber laminate ( $\theta=45)$

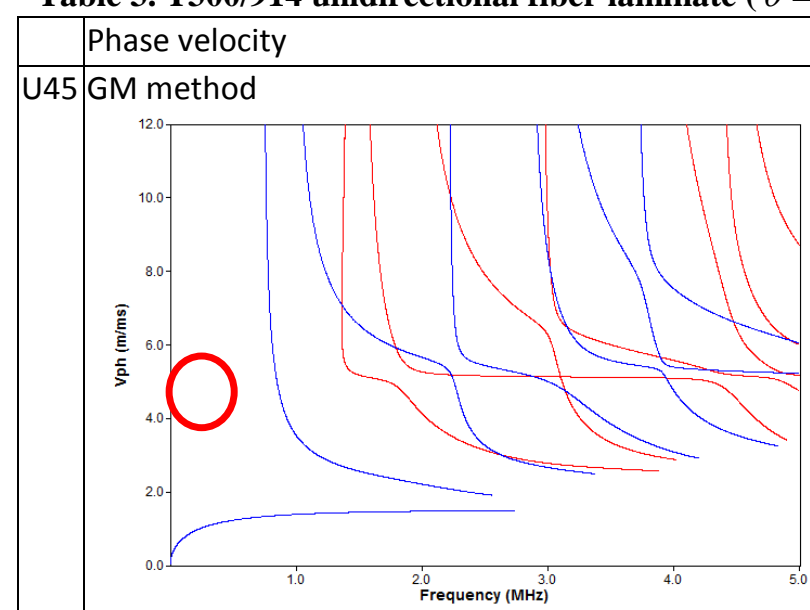

SAFE method

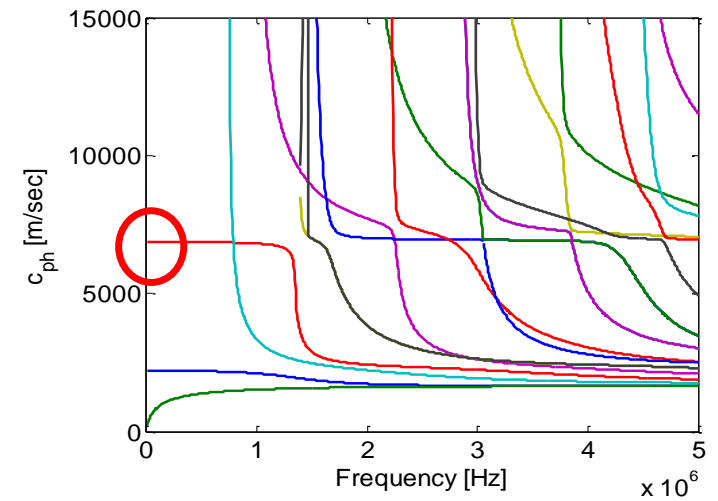

For completeness, we recall DISPERSE results reported in [16] pp. 157.

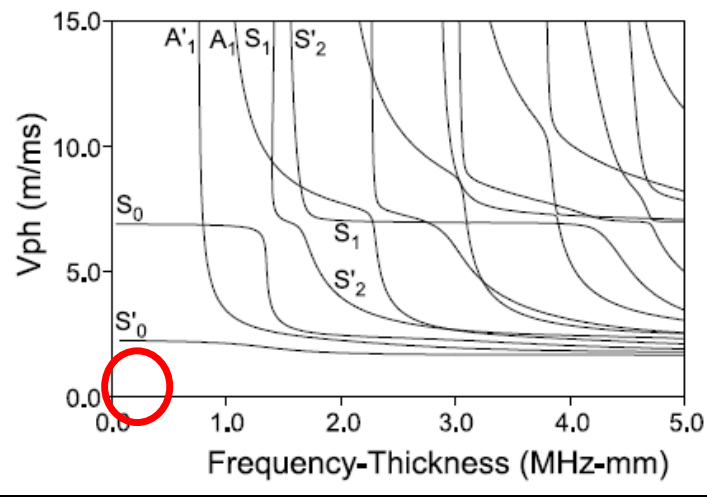

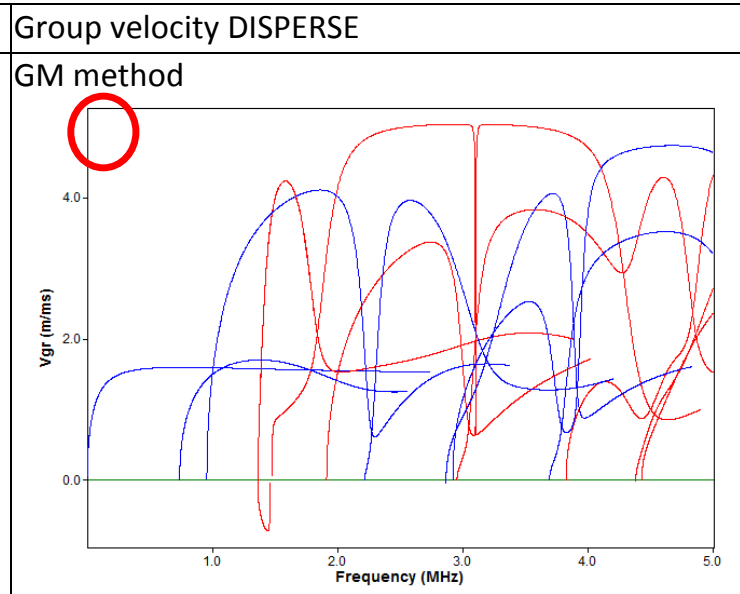

SAFE method

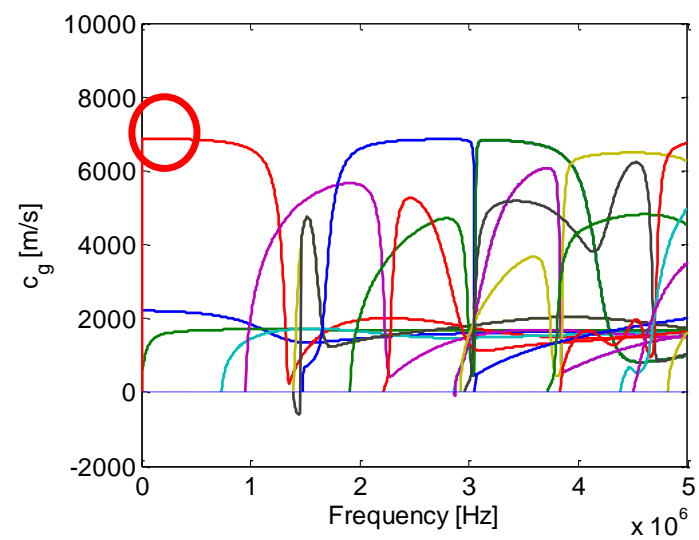

DISPERSE, Ref. [16], pp. 157

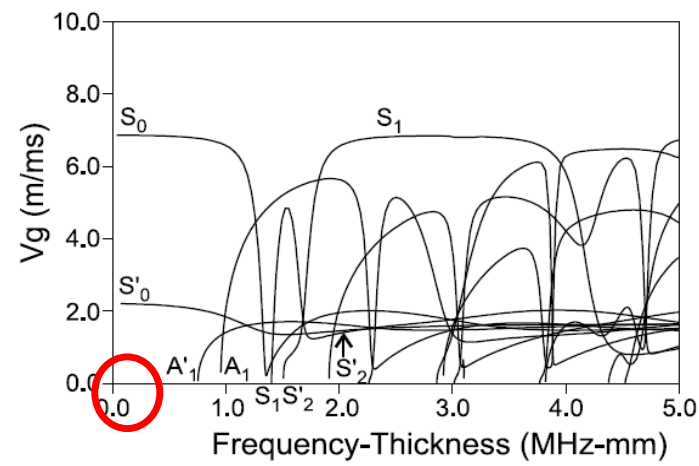

Results of $\theta=45$, (Table 3) indicate a missing $\mathrm{A}_{0}$ from $\mathrm{GM}$ run. On the other hand, the corresponding dispersion curves reported in literature [16] showed a missing $\mathrm{A}_{0}$. 
Table 4. T300/914 unidirectional fiber laminate $(\theta=90)$

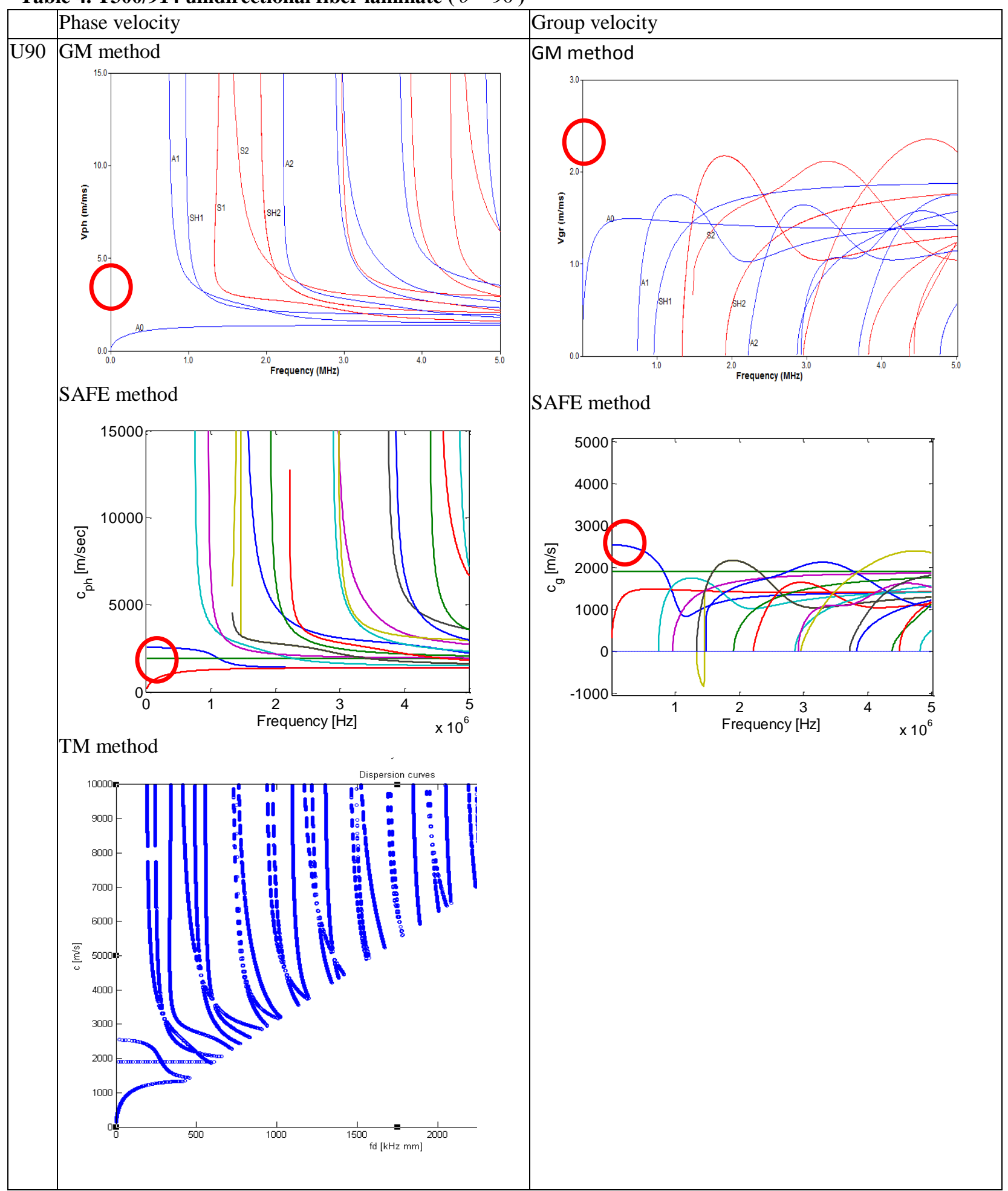

Table 4 shows good correlation between the three methods for U90 case. However, more investigation is needed for TM method, to fix the frequency axis mismatch. As a general note, all cases are normalized to be 1-mm thick laminates. 
A comparison between LISA method, and DISPERSE (i.e. GM) is reported in [25]. The material used is unidirectional IM7 Cycom 977-3 multilayer unidirectional laminate with $1.5 \mathrm{~mm}$ thick. The authors compared $\mathrm{A}_{0}$ mode using group velocity dispersion curves.

\section{B. Cross-ply laminated composites}

In this subsection, we study two cases: [0/90] laminate with each layer is $0.5 \mathrm{~mm}$ thick and $[0 / 90]_{\mathrm{s}}$ with each layer equals $0.25 \mathrm{~mm}$ thick. The material is the same T300/914 used before. In this section, we use Equivalent Matrix method as well for comparison.

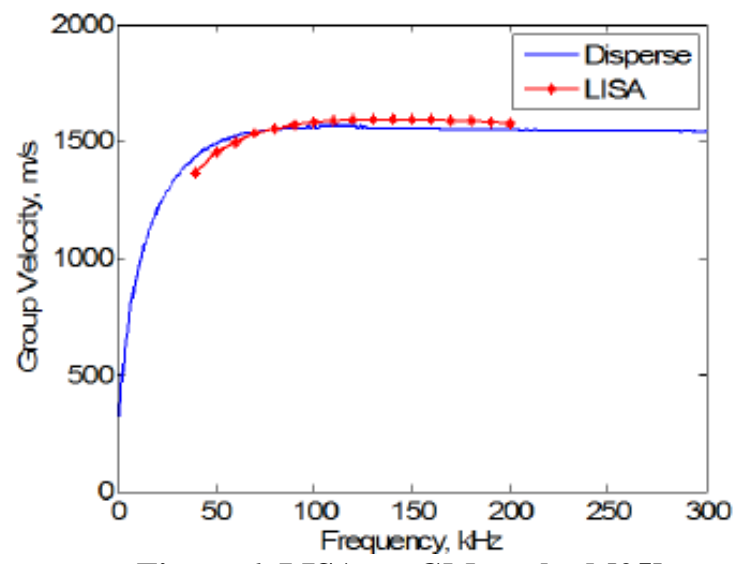

Figure 6. LISA vs. GM method [25]

Table 5. T300/914 cross ply fiber laminate, [0/90]

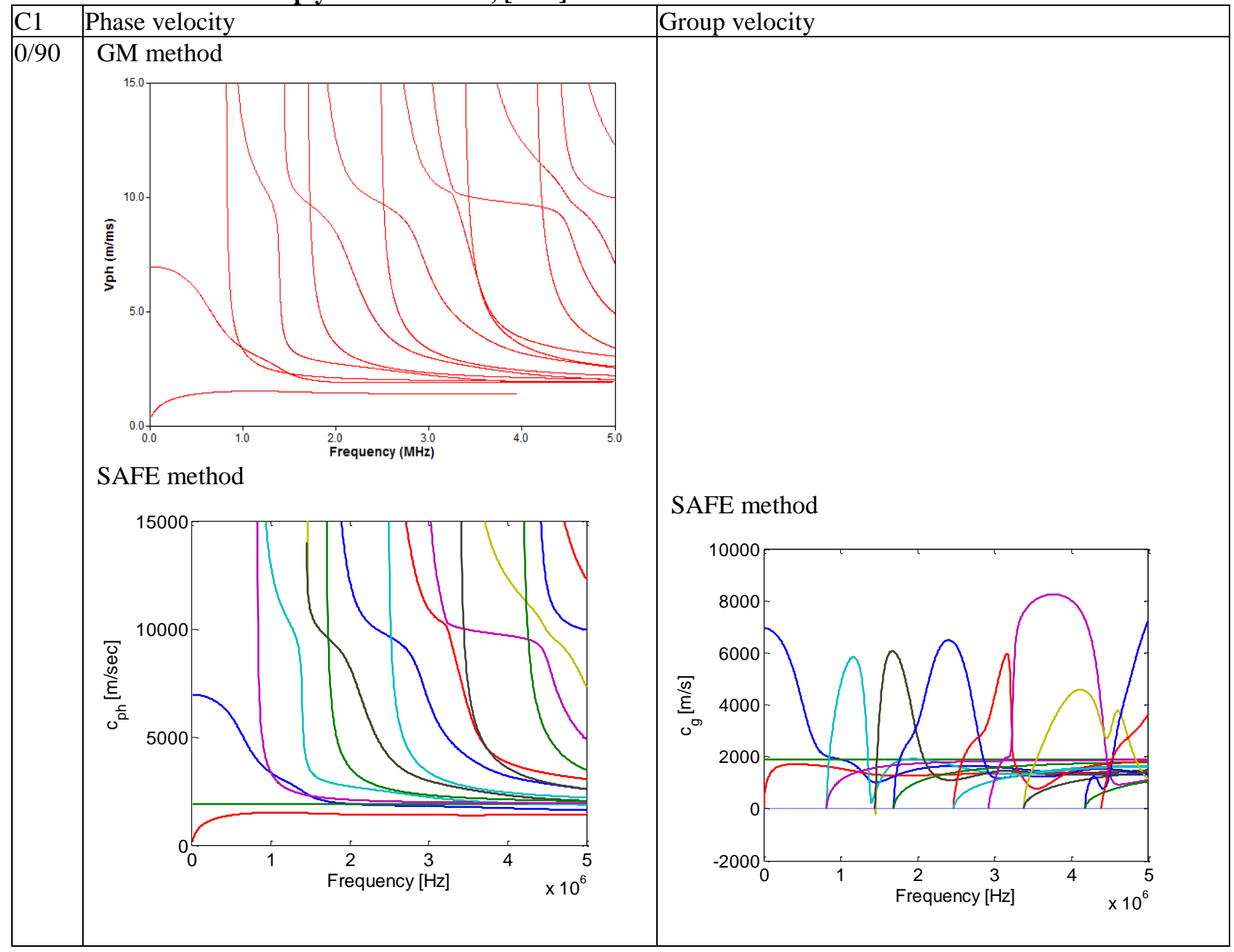

Table 5 shows GM and SAFE comparisons. In the next page, Table 5 cont'd shows phase velocity for both TM and EM methods. it is concluded that EM can just give a rough estimation, especially for the cases of few number of layers, but it is noticed that $S_{0}$ speed at very low frequency is almost $7000 \mathrm{~m} / \mathrm{s}$ in all method. GM and SAFE agree very well. TM method has the same trend with the same glitch in frequency axis, i.e. the first mode after the three $\mathrm{S}_{0}, \mathrm{~A}_{0}$, and $\mathrm{SH}_{0}$ has a cut off frequency around $1 \mathrm{MHz}$; while, for TM method it is shown less than $500 \mathrm{kHz}$. 


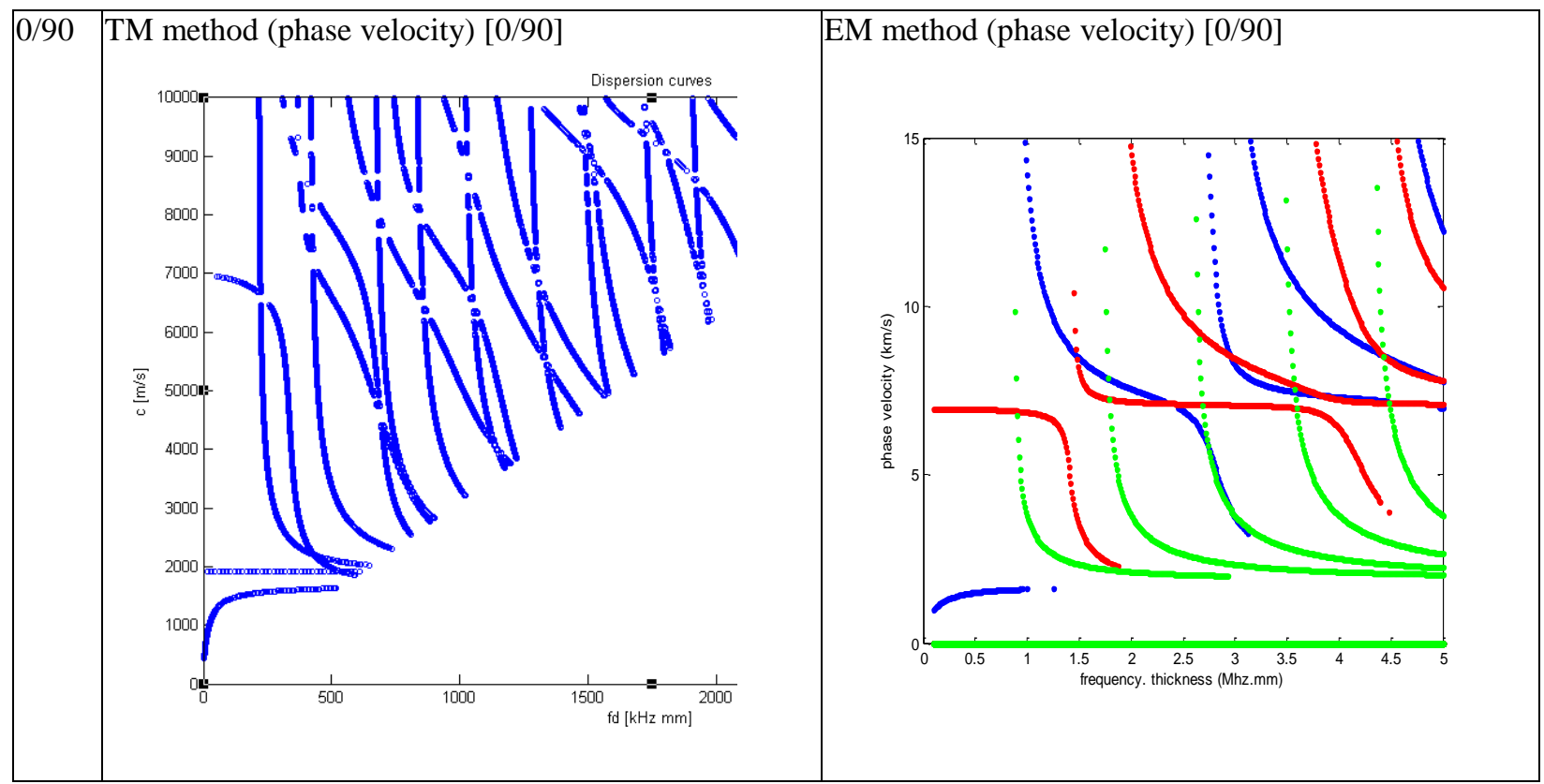

Finally, this subsection ends by $[0 / 90]_{\text {s }}$ case study of T300/914 CFRP, (Table 6). EM will show similar result as the one for [0/90].

Table 6. T300/914 cross ply fiber laminate, $[0 / 90]_{\mathrm{s}}$

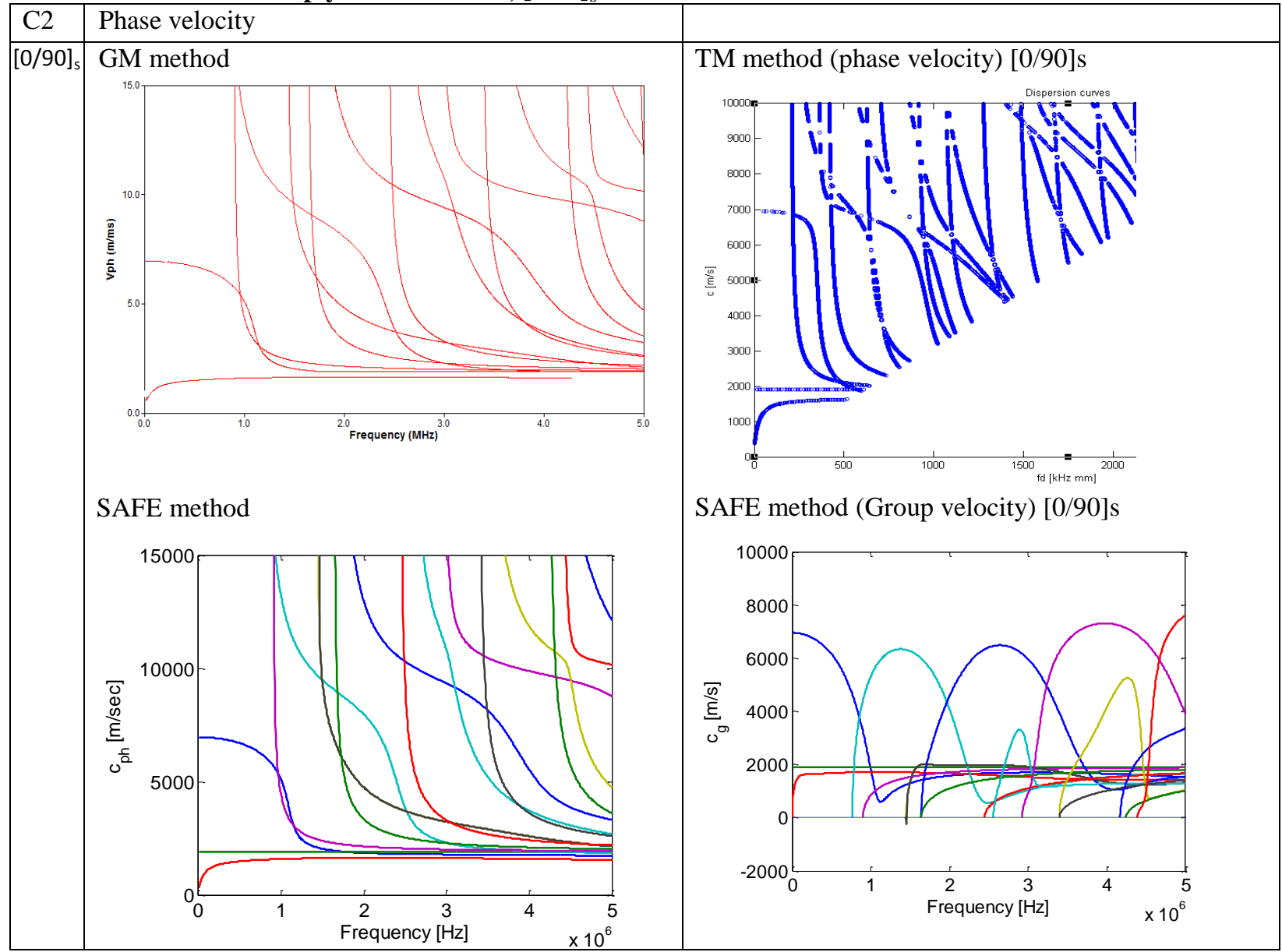

American Institute of Aeronautics and Astronautics 


\section{Quasi isotropic composite Laminate}

The material used for this case study is T800/924 CFRP, following the example in [16], pp. 158. The laminate total stack orientation is defined as $[+45 /-45 / 0 / 90]_{s}$. The layer material properties are

$$
E_{11}=161 \mathrm{GPa}, E_{33}=9.25 \mathrm{GPa}, G_{13}=6 \mathrm{GPa}, v_{13}=0.34, v_{23}=0.41 \quad, \rho=1500 \mathrm{~kg} / \mathrm{m}^{3}
$$

And the corresponding stiffness coefficients are

$$
\begin{aligned}
& C_{11}=164.7 \mathrm{GPa}, C_{12}=C_{13}=5.45 \mathrm{GPa}, C_{22}=C_{33}=11.3 \mathrm{GPa} \\
& C_{23}=4.7 \mathrm{GPa}, C_{44}=3.3 \mathrm{GPa} \quad, C_{55}=C_{66}=6 \mathrm{GPa}
\end{aligned}
$$

Table 7. t800/924 Quasi isotropic composite laminate $[+45 /-45 / 0 / 90]_{\mathrm{s}}$

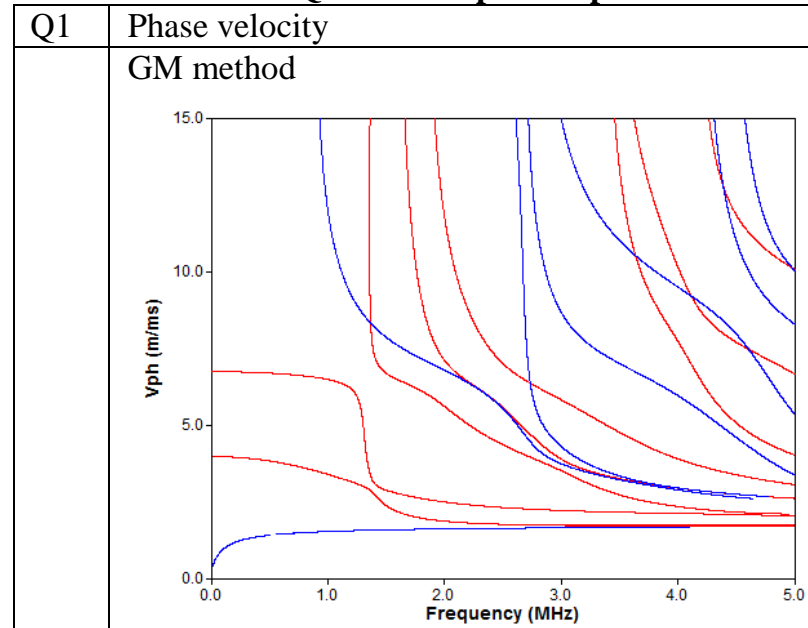

SAFE method

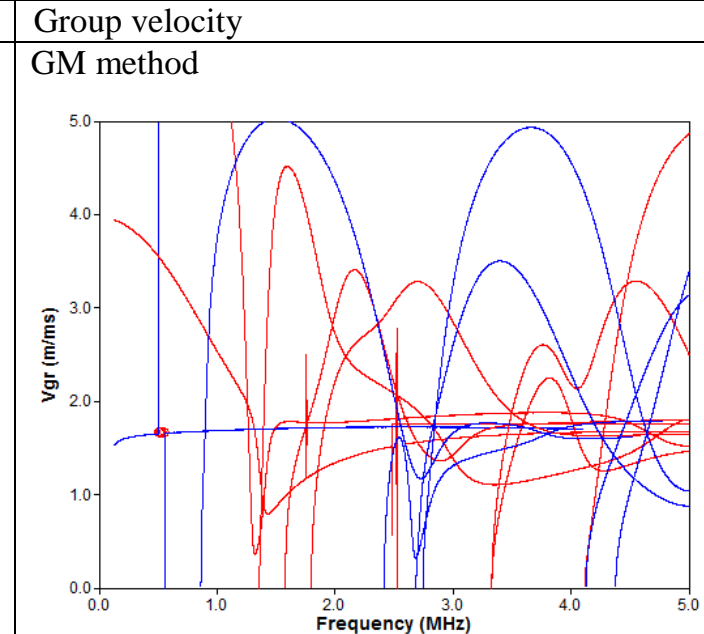

SAFE method
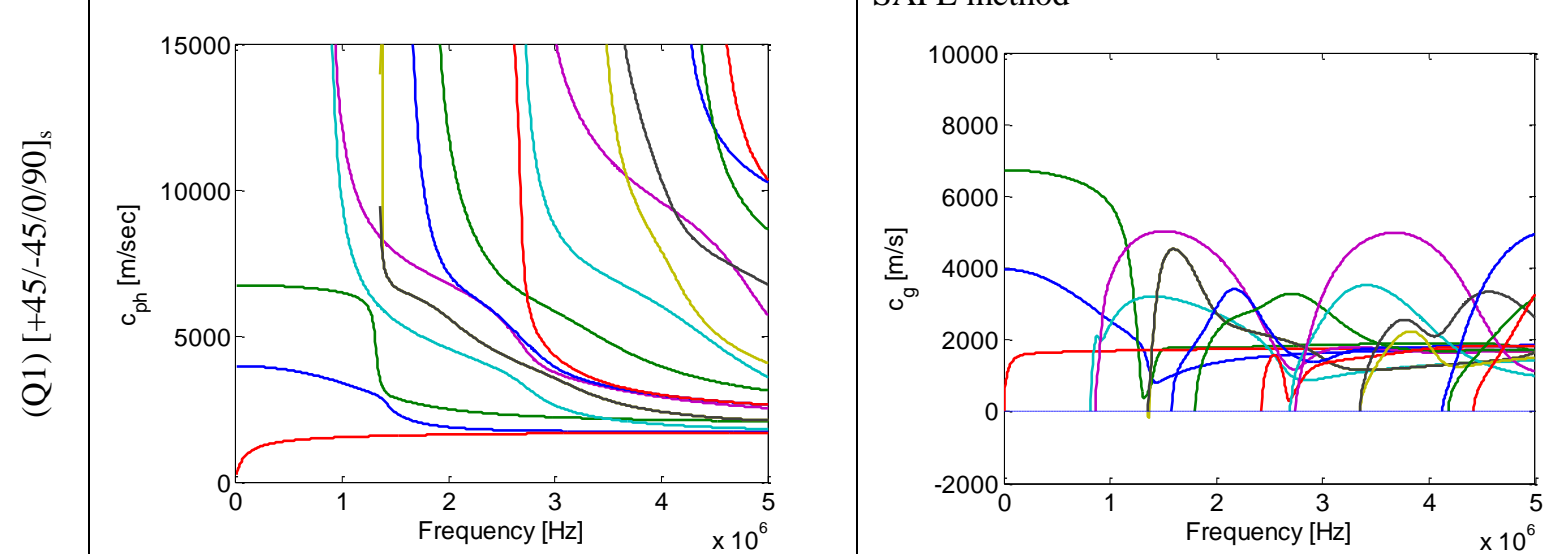

TM method

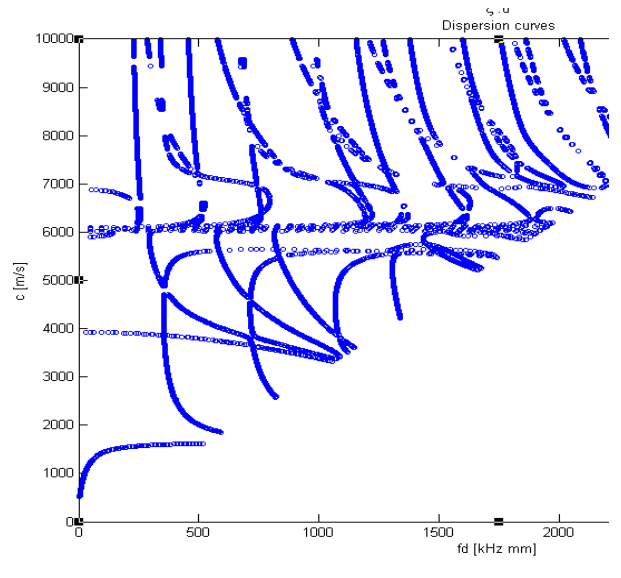




\section{Experimental and Finite Element Simulation Results}

\section{A. Experimental setup}

The structure under investigation is a Norplex-Micarta 'NP130' fiberglass plate consisting of a woven glass fabric substrate combined with an epoxy resin system. This material is commonly used in naval industry. All the plies are oriented in the same direction, so the resulting laminate is symmetric and presented two main directions with very similar elastic properties at 0 and 90 degree. The plate dimension is $850 \times 850 \times 3.2 \mathrm{~mm}^{3}$ and the material density is $1960 \mathrm{~kg} / \mathrm{m}^{3}$. For the modeling of the dispersion curves of Lamb modes, equivalent matrix method [24] was used to find the total stiffness matrix [C] of our material [26]

$$
C=\left[\begin{array}{cccccc}
21.2 & 3 & 5.7 & 0 & 0 & 0 \\
21.2 & 20.2 & 3.7 & 0 & 0 & 0 \\
5.7 & 3.7 & 13.4 & 0 & 0 & 0 \\
0 & 0 & 0 & 5.8 & 0 & 0 \\
0 & 0 & 0 & 0 & 5.9 & 0 \\
0 & 0 & 0 & 0 & 0 & 5.7
\end{array}\right] G P a
$$

A large number of piezoelectric wafer active sensors (PWAS) disks (Steminc SM412, 7mm diameter disks) are used for Lamb wave propagation analysis, involving various angular directions. The PWAS network bonded on the glass laminate is described on Figure 7. We performed the experimental acquisitions; from 1 to $300 \mathrm{kHz}$ step $6 \mathrm{kHz}$, using PWAS \#0 as actuator, and the other PWAS disks as sensors. As exciting signals we used 3 sine cycles with Hanning window, having maximum amplitude 100 Volts.

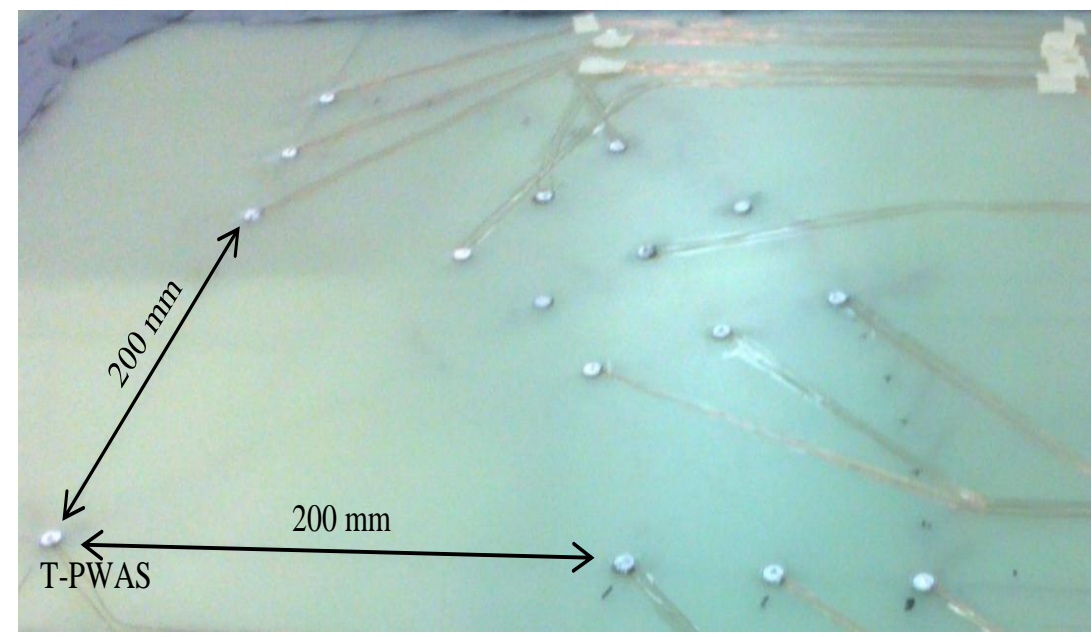

Figure 7. Picture the network of piezolectric wafer active sensor bonded on the NP130 specimen

\section{B. Dispersion curve in GFRP}

Figure 8 shows the experimental dispersion curve for the propagation path at 0 degree. As we can observe, only two modes are present, A0 and S0 mode. Moreover the velocities of these modes have an acceptable match with the dispersion curve calculated analytically. The SH0 mode is not detected in this study. 


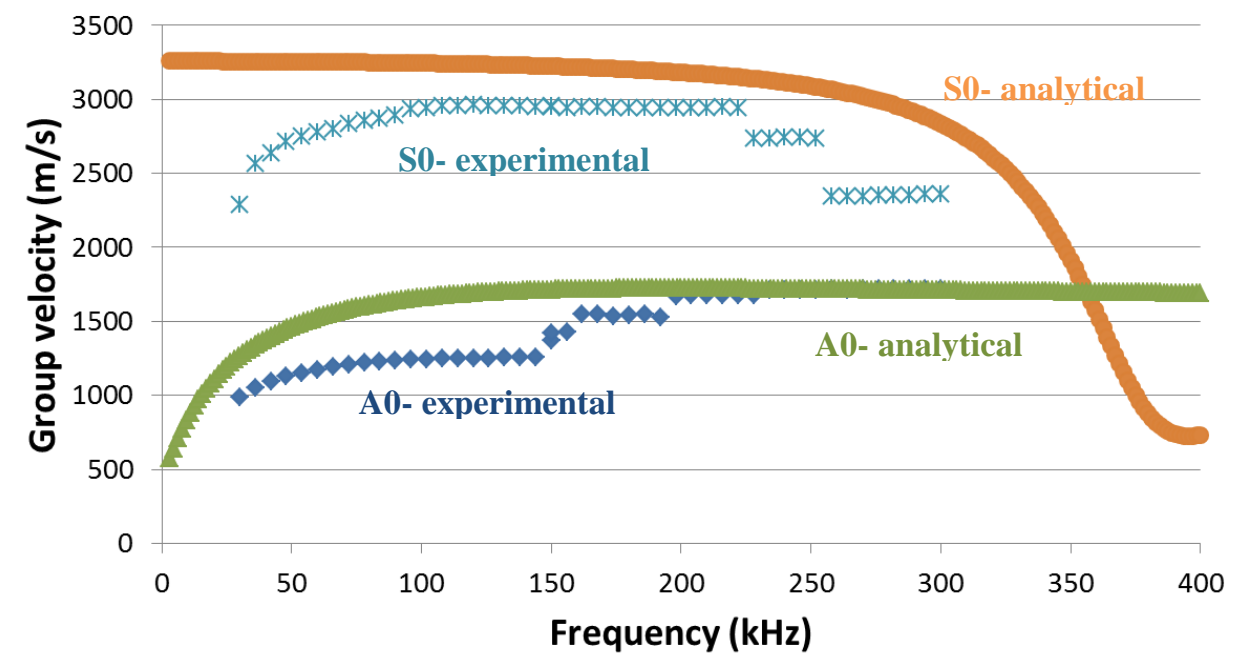

Figure 8. Comparison between experimental and analytical dispersion curves for the propagation path at 0 degree

An example for quasi isotropic plate [(0/45/90/-45 $\left.)_{2 \mathrm{~s}}\right]$ was reported in Ref. [27], we include it here as an example for CFRP materials. The layer material is A534/AF252, same stiffness properties of T300/5208. Experimental results were compared with the analytical TM after calculating group velocity curves from the phase velocity ones (Figure 9)

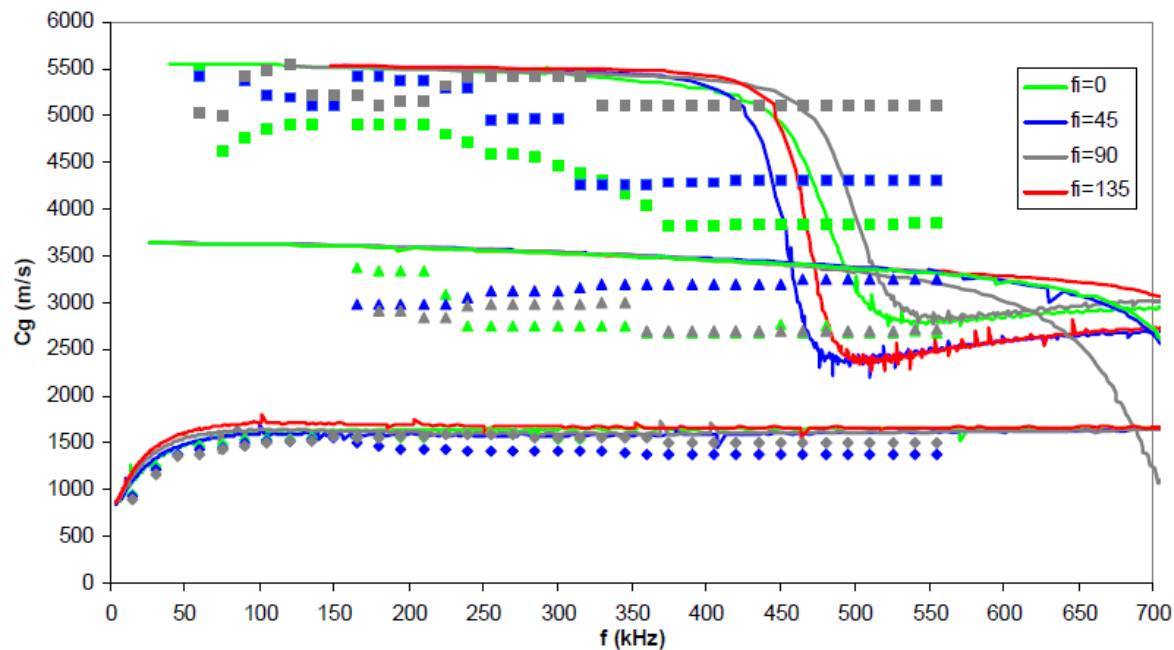

Figure 9. Group velocities for a quasi-isotropic plate. (Solid lines) theoretical values and (dots) experimental values [27].

\section{FEM model}

The aim of the numerical studies is to systematically investigate the robustness of the proposed Lamb-wavebased methodology for anisotropic composite laminates. The finite element (FEM) is employed to simulate Lamb wave propagation. The effectiveness of conventional finite element modeling of elastic waves propagating in structural components has been shown in the past. The case of Lamb waves in free isotropic plates is a classic example [28, 29]. The commercial software used in the present study, ABAQUS, is based on a central difference method [30]. In preliminary studies [31, 32], we investigated how the group velocities of the S0 and A0 waves vary with mesh density (nodes per wavelength) $N=\lambda / L$, where $\lambda$ is the wavelength and $L$ is the size of the element for an isotropic structure. For a 1-D wave propagation problem, we choose a mesh size of $0.1 \mathrm{~mm}$ corresponding to $N=76$, which mitigated a reasonable accuracy with an acceptable computation time. However this small mesh size 
of $0.1 \mathrm{~mm}$ is not practical for 2-D wave propagation because of considerable long time of calculation. In our 2-D case, we choose a mesh size of $0.5 \mathrm{~mm}$ which gives an acceptable computation time and reasonable accuracy. The fundamental anti-symmetrical mode (A0) is preferable and more sensitive to damage because its wavelength is shorter than that of the S0 mode at the same frequency. However, the A0 mode exhibits more dispersion at low frequencies. The FEM simulation of the A0 mode requires fine spatial discretization with substantial computational cost for the sake of the short wavelength. In contrast, the mode shapes of the S0 mode are simpler and the stresses are almost uniform throughout the thickness of the plate at low values of the frequency-thickness product. For these reason, the two modes $\mathrm{S} 0$ and $\mathrm{A} 0$ were selected in this study to compare with the experimental results.

The use of multi-physics finite element method (MP-FEM) allows us to directly apply the excitation voltage at the transmitter PWAS (T-PWAS) and directly record the capture signal at the receiver PWAS (R-PWAS). We used the ABAQUS implicit code to simulate the generation of Lamb waves in a 3.2-mm laminate glass fiber plate with surface mounted 7-mm round PWAS placed as shown in Figure 7. The plate was discretized with S4R shell elements of size $1-\mathrm{mm}$ in the $x y$ plane (mesh density comprised between $N=23$ and $N=8$ elements per wavelength). Each laminate is composed of three integration points. The PWAS transducers were discretized with the C3D8E piezoelectric element. We modeled the electric signal recorded at the R-PWAS due to an electric excitation applied to the T-PWAS which generated ultrasonic guided waves travelling through the plate. The piezoelectric material properties were assigned to the PWAS as described in [33].

Figure 10 shows the MP-FEM simulation electric signal measured at R-PWAS placed 200-mm from the TPWAS. The predicted signal using the damping coefficients is compared with the experimental results. At the excitation frequency of $f=150 \mathrm{kHz}$, two guided wave modes are present, S0, and A0. The A0 mode is considerably slower than the S0 mode and its magnitude is almost 0 compared to the magnitude of the S0 mode. In addition, a good magnitude match is obtained for the first part of the packet for the S0 mode between the experimental and the MP-FEM results. However, for these mass and stiffness proportional coefficient $\alpha_{M}=15,000 \mathrm{rad} / \mathrm{s}$ and $\beta_{K}=5.10^{-8} \mathrm{~s} / \mathrm{rad}$, the MP-FEM magnitude for the A0 mode is smaller than the experiment value, i.e. the damping coefficient is too big to simulate the experimental value of the A0 mode.

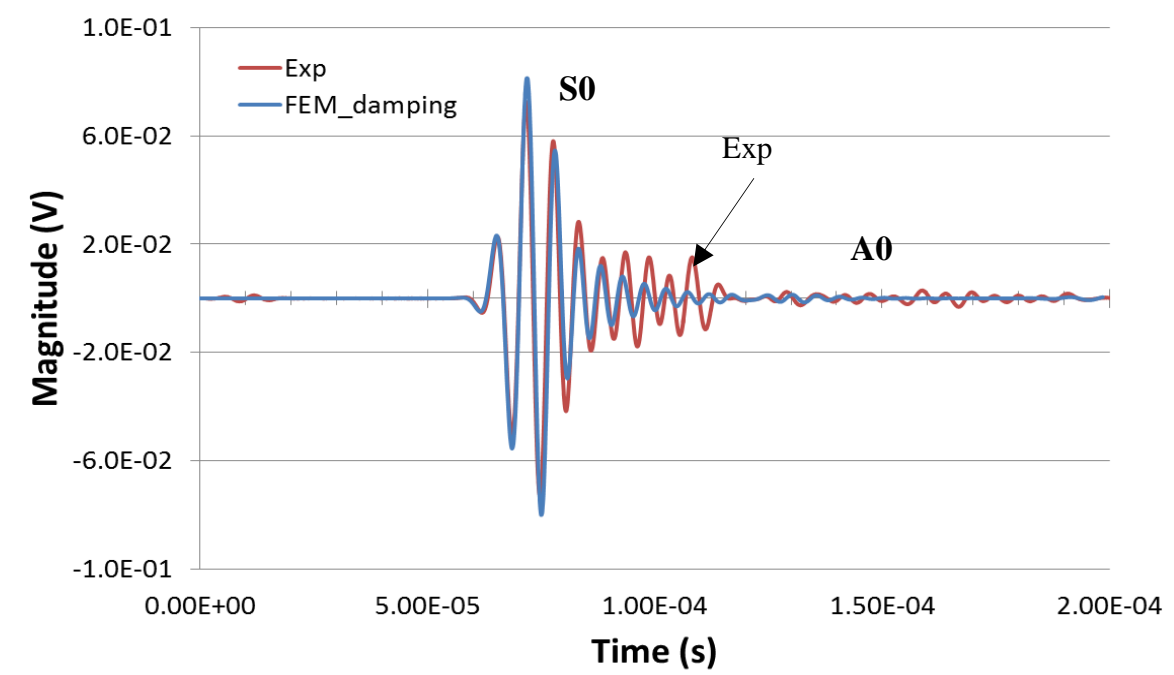

Figure 10. Received signal at $200 \mathrm{~mm}$ from the transmitter PWAS at $150 \mathrm{kHz}$ between the experimental and the FEM results with damping.

\section{Conclusion}

Different algorithms were evaluated for calculating dispersion wave speeds in composites. The paper covered briefly the mathematical formulation of each method with more details on the transfer matrix method and efforts for generating a stable robust algorithm. Transfer matrix method is a reliable technique for wave propagation analysis in layered media; its advantage is that it condenses the multi-layered system into four equations (for the case of decoupled SH waves) or six equations relating the boundary conditions at the first and the last interfaces. It 
eliminates all other intermediate interfaces; which save a lot in terms of computational time and complexity. Hence, TM method is favorable. One drawback TM method suffers is its numerical instability of the solution at large frequency-thickness products. There have been many publications proposing reformulation of TM equations to avoid this problem. The method is based on using stiffness matrix (SM) instead of TM; this is done by re-arranging terms of the TM such that displacements at both the top and the bottom of the a layer are in a single column matrix, and similarly for stresses.

Global matrix combines stresses and displacements at the boundaries of each layer with the whole system boundary conditions and assembles them in one single matrix. Global matrix has the advantage that it remains stable at high frequency-thickness products. The disadvantage is that the global matrix ends to be a large matrix for laminates with large numbers of layers. Semi Analytical Finite Element (SAFE) discretizes the structure cross section allowing different cross sections to be analyzed, because of the finite element discretizing in cross section. In the same time it solves analytically for wave propagation direction; which makes it more efficient in terms of computational time and memory than a complete FEM. In general the material is defined in FEM by stiffness matrix; this makes SAFE method a very straight forward for application for anisotropic materials. SAFE is becoming popular for analyzing guided wave propagation in composites. Local Interaction Simulation Approach (LISA) discretizes the system into a lattice like finite difference method and its formulation is based on elastodynamic equations. The advantage of LISA appears when discontinuities or changes needed to be applied to the material properties; those changes are treated by simply modifying the properties of the lattice at the corresponding locations. Equivalent Matrix (EM) is very quick and reliable approach for analyzing cross ply laminates due to the fact that transformation matrix between 0 and 90 degrees is straight forward and can be done manually.

The paper presented case studies for (a) unidirectional composites with wave propagation along different angles and GM had very good agreement with SAFE method. Our TM tool is still under development and it showed similar results in terms of speeds values; however, cut off frequencies were not correct. Next, we presented case studies on (b) cross-ply laminates; in this case, results were compared with EM method as well. Trends seemed to agree with differences along frequency axes. Finally, (c) the paper presented comparisons on quasi-isotropic laminates, and it showed very good agreement between GM and SAFE methods.

Finally, the paper ended by experimental and finite element studies on GFRP woven composite sample. Piezoelectric wafer active sensors (PWAS) transducers were used for pitch catch experiments using three tone burst excitation signals. Experimental dispersion group velocities showed good agreement with the theory. In Multiphysics finite element model, we used shell elements for the plate and coupled field element for the transducer; this allowed simulation of the input excitation and output response in voltage. Rayleigh damping was considered in the model and careful selection of damping coefficients is important for simulating actual experimental response.

As an overall conclusion, analytical based methods are favorable compared to FEM. Although many analytical methods incorporate numerical root finding algorithms, but they are more efficient than FEM in computational resources; with the major key fact that FEM can model complex geometries. This limitation in analytical methods is no more an issue after developing semi analytical finite element tools which can model arbitrary cross sections. New methods need to be designed for even complex sandwich composites with complex filling structures (e.g. honeycomb).

\section{Acknowledgments}

Support by Air Force Office of Scientific Research, \#FA9550-11-1-0133, Dr. David Stargel, Program Manager; is thankfully acknowledged. The authors would like to thank Prof. Mike Lowe from Imperial College of London, Prof. Ivan Bartoli from Drexel University, and Prof. Alessandro Marzani from University of Bologna for their help in providing some dispersion curves simulations using computer packages they developed. Thanks to Prof. Stanislav Rokhlin for his invaluable comments.

\section{References}

${ }^{1}$ Rose JL. Ultrasonic Waves in Solid Media. New York: Cambridge University Press; 1999.

${ }^{2} \mathrm{Su}$ Z, Ye L, Lu Y. Guided Lamb waves for identification of damage in composite structures: A review. J. of Sound and Vibration. 2006;295(3-5):753-780.

${ }^{3}$ Giurgiutiu V, Santoni G. Structural Health Monitoring of Composite Structures with Piezoelectric-Wafer Active Sensors. AIAA Journal. $2011 ; 49(3): 565-581$. 
${ }^{4}$ Graff KF. Wave motion in elastic solids. Dover Publications Inc.; 1991.

${ }^{5}$ Giurgiutiu V. Structural Health Monitoring with Piezoelectric Wafer Active Sensors. Elsevier Academic Press; 2008.

${ }^{6}$ Viktorov IA. Rayleigh and Lamb waves - physical theory and applications. New York: Plenum Press; 1967.

${ }^{7}$ Thomson W. Transmission of elastic waves through a stratified solid medium. journal of Applied Physics. 1950;21:89-93.

${ }^{8}$ Haskell N. The dispersion of surface waves on multi-layered media. Bulletin of the Seismological Society of America. 1953;43(1):17-34.

${ }^{9}$ Lowe M. Matrix techniques for modeling ultrasonic waves in multilayered media,"Ultrasonics. Ferroelectrics and Frequency Control, IEEE Transactions. 1995;42(4):525-542.

${ }^{10}$ Nayfeh AH. Wave Propagation in Layered Anisotropic Media. New York: Elsevier; 1995.

${ }^{11}$ Santoni, Giola. Fundamental Studies in the Lamb-Wave Interaction Between Piezoelectric Wafer Active Sensor and Host Structure During Structural Health Monitoring. Electronic Theses and Dissertations. Dissertation, University of South Carolina; 2010.

${ }^{12}$ Jones R. Mechanics of composite materials. London: Taylor \& Francis; 1999.

${ }^{13}$ Rokhlin S, Chimenti D, Nagy P. Physical Ultrasonics of Composites. Oxford University Press; 2011.

${ }^{14}$ Wang L, Rokhlin SI. Stable reformulation of transfer matrix method for wave propagation in layered anisotropic media. J. Ultrasonics. 2001;39:407-418.

${ }^{15}$ Knopoff L. A matrix method for elastic wave problems. Bulletin of the Seismological Society of America. 1964;54:431438.

${ }^{16}$ Pavlakovic B, Lowe M. DISPERSE Manual [Internet]. 2003.

${ }^{17}$ Gavric L. Computation of Propagative Waves in Free Rail Using a Finite Element Technique. Journal of Sound and Vibration. 1995;185(3):531-543.

${ }^{18}$ Sorohan S, Constantin N, Gavan M, Anghel V. Extraction of dispersion curves for waves propagating in free complex waveguides by standard finite element codes. Ultrasonics. 2011;51:503-515.

${ }^{19}$ Hayashi T, Song WJ, Rose JL. Guided wave dispersion curves for a bar with an arbitrary cross-section, a rod and rail example. Ultrasonics. 2003;41:175-183.

${ }^{20}$ Bartoli I, Marzani A, Lanza di Scalea F, Viola E. Modeling Wave Propagation in Damped Waveguides of Arbitrary Crosssection. J. Sound \& Vibration. 2006;295:685-707.

${ }^{21}$ Delsanto PP, Schechter RS, Mignogna RB. Connection machine simulation of ultrasonic wave propagation in materials III: The three-dimensional case. Wave Motion. 1997;26(4):329-339.

${ }^{22}$ Ruzzene M, Jeong SM, Michaels TE, Michaels JE, Mi B. Simulation and measurement of ultrasonic waves in elastic plates using laser vibrometry. In: Thompson DO, Chimenti DE, editors. Review of progress in Quantitative Non-destructive; 2005; Green Bay, Wisconsin. p. 172-179.

${ }^{23}$ Nadella K, Cesnik C. Numerical simulation of wave propagation in composite plates. In: Proceedings of SPIE 8348, 83480L; 2012.

${ }^{24}$ Monnier T. Lamb Waves-based Impact Damage Monitoring of a Stiffened Aircraft Panel using Piezoelectric Transducers. J. Int. Mat. Sys. and Structures. 2006;17:411-421.

${ }^{25}$ Nadella K, Cesnik C. Simulation of Guided Wave Propagation in Isotropic and Composite Structures using LISA. In: 53rd AIAA/ASME/ASCE/AHS/ASC Structures, Structural Dynamics and Materials Conference; 2012.

${ }^{26}$ Pollock P, Yu L, Sutton MA, Guo S, Majumdar P, Gresil M. Full-Field Measurements for Determining Orthotropic Elastic Parameters of Woven Glass-Epoxy Composites Using Off-Axis Tensile Specimens. Experimental Techniques. 2012.

${ }^{27}$ Santoni G, Chrysochoidis NA, Giurgiutiu V, Saravanos DA. Analytical and Experimental Evaluation of Piezoelectric Wafer Active Sensors Performances for Lamb Waves based Structural Health Monitoring in Composite Laminates. In: Proc. of SPIE, Non Destructive Evaluation; 2007; March 19-22, San Diego, CA. p. 6532-22.

${ }^{28}$ Alleyne DN, Cawley P. A 2-dimensional Fourier transform method for the quantitative measurement of Lamb modes.; 1990. p. 1143,1146.

${ }^{29}$ Moser F, Jacobs LJ, Qu J. Modeling elastic wave propagation in waveguides with the finite element method. NDT \& E International. 1999;32(4):225-234.

${ }^{30}$ ABAQUS. Analysis User's Manual, 6-9.2 ed. 2008.

${ }^{31}$ Gresil M, Shen Y, Giurgiutiu V. Benchmark problems for predictive fem simulation of 1-D and 2-D guided waves for structural health monitoring with piezoelectric wafer active sensors. In: Review of Progress in Quantitative Non-destructive Evaluation; 2011. p. 1835-1842.

${ }^{32}$ Gresil M, Shen Y, Giurgiutiu V. Predictive modeling of ultrasonics SHM with PWAS transducers. In: 8th International Workshop on Structural Health Monitoring; 2011; Stanford, CA.

${ }^{33}$ Gresil M, Yu L, Giurgiutiu V, Sutton M. Predictive modeling of electromechanical impedance spectroscopy for composite materials. Structural Health Monitoring. 2012;11(6):671-683.

${ }^{34}$ Gresil M, Giurgiutiu V. Guided wave propagation in composite laminate material using piezoelectric wafer active sensor. RAeS Aeronautical Journal. accepted for publication March 2013. 